\title{
Robust Backlash Estimation for Industrial Drive-Train Systems-Theory and Validation
}

Papageorgiou, Dimitrios; Blanke, Mogens; Niemann, Hans Henrik; Richter, Jan H.

Published in:

IEEE Transactions on Control Systems Technology

Link to article, DOI:

10.1109/TCST.2018.2837642

Publication date:

2019

Document Version

Peer reviewed version

Link back to DTU Orbit

Citation (APA):

Papageorgiou, D., Blanke, M., Niemann, H. H., \& Richter, J. H. (2019). Robust Backlash Estimation for Industrial Drive-Train Systems-Theory and Validation. IEEE Transactions on Control Systems Technology, 27(5), 18471861. https://doi.org/10.1109/TCST.2018.2837642

\section{General rights}

Copyright and moral rights for the publications made accessible in the public portal are retained by the authors and/or other copyright owners and it is a condition of accessing publications that users recognise and abide by the legal requirements associated with these rights.

- Users may download and print one copy of any publication from the public portal for the purpose of private study or research.

- You may not further distribute the material or use it for any profit-making activity or commercial gain

- You may freely distribute the URL identifying the publication in the public portal 


\title{
Robust Backlash Estimation for Industrial Drive-Train Systems-Theory and Validation
}

\author{
Dimitrios Papageorgiou $^{\circledR}$, Mogens Blanke ${ }^{\circledR}$, Senior Member, IEEE, \\ Hans Henrik Niemann ${ }^{\circledR}$, and Jan H. Richter ${ }^{\circledR}$
}

\begin{abstract}
Backlash compensation is used in modern machinetool controls to ensure high-accuracy positioning. When wear of a machine causes deadzone width to increase, high-accuracy control may be maintained if the deadzone is accurately estimated. Deadzone estimation is also an important parameter to indicate the level of wear in a machine transmission, and tracking its development is essential for condition-based maintenance. This paper addresses the backlash estimation problem using sliding-mode and adaptive estimation principles and shows that prognosis of the development of wear is possible in both theory and practice. This paper provides the proof of asymptotic convergence of the suggested estimator, and it shows how position offset between motor and load is efficiently utilized in the design of a very efficient estimator. The algorithm is experimentally tested on a drive-train system with the state-of-the-art Siemens equipment. The experiments validate the theory and show that expected performance and robustness to parameter uncertainties are both achieved.
\end{abstract}

Index Terms-Adaptive deadzone estimation, backlash estimation, experimental validation, machine tools, mechanical drive train, nonlinear parameterization, parameter estimation, robustness analysis, sliding-mode observer (SMO).

\section{INTRODUCTION}

D EVELOPING backlash in coupling equipment due to wear is one of the main reasons for performance degradation in machine-tool systems. Since high-accuracy tool positioning is fundamental for maintaining the workpiece tolerances, backlash compensation solutions are used in nearly all modern computer numerical control algorithms. As such, knowledge of the deadzone angles in advance is essential for integrating backlash compensation in the position servo loops of the machine drive motors. In this context, online estimation methods may facilitate an automatic compensation solution for developing backlash and also provide valuable information for prognosis and equipment lifetime.

Manuscript received December 20, 2017; accepted May 10, 2018. Manuscript received in final form May 14, 2018. This work was supported by Siemens AG. Recommended by Associate Editor S. Pirozzi. (Corresponding author: Dimitrios Papageorgiou.)

D. Papageorgiou, M. Blanke, and H. H. Niemann are with the Department of Electrical Engineering, Technical University of Denmark, 2800 Kongens Lyngby, Denmark (e-mail: dimpa@elektro.dtu.dk; mb@ elektro.dtu.dk; hhn@elektro.dtu.dk).

J. H. Richter is with the Digital Factory Division, Siemens AG, D-90475 Nuremberg, Germany (e-mail: janrichter@siemens.com).

Color versions of one or more of the figures in this paper are available online at http://ieeexplore.ieee.org.

Digital Object Identifier 10.1109/TCST.2018.2837642
The backlash phenomenon in various mechanical systems has been extensively studied over the past three decades. Indirect backlash estimation methods pertain to identifying torques and accelerations of the system parts that are affected by the backlash and from these signals drawing conclusions on the width of the deadzones. A characteristic example is the work in [1], where backlash estimation in a gearing system was done via calculation of the speed change of the driving part of the gear. An extended Kalman filter was employed in [2] and [3] for estimating the backlash torque in a two-mass motor arm and an automotive powertrain. The backlash function parameters were identified offline based on this estimated torque. Hågglund [4] used describing functions to model the effects of backlash in a closed-loop motion system. He presented an online calculation making use of a static relation between backlash model parameters and controller gains. Optimization techniques for offline identification of backlash torque were employed in [5] for cascaded linear systems and in [6] for a vehicle drive-line system, where position, velocity, and torque measurements were used. The backlash torque was modeled with a "contact"/"noncontact" approach in [7]. The "noncontact" torque was modeled by a differentiable function of deadzone width and assessed the backlash amplitude indirectly through "noncontact" torque estimation in a nonlinear observer. Experimental validation was presented in [8] using a sliding-mode observer (SMO).

In most of the previous studies, backlash was described as the resulting torque when contact of the two moving parts of the coupling takes place. Direct estimation of the deadzone angle has mainly been addressed as an offline identification problem with methods that are valid for perturbations around a linearization point of the system [2], [3], [5], [6]. In our prior work [9], we proposed a smooth backlash model based on variable shaft stiffness and outlined the basic ideas of a cascaded architecture for backlash estimation based on slidingmode and adaptive principles along with simulations.

This paper extends our previous work by presenting a complete theoretical framework for the design of the deadzone angle estimator. Specifically, the varying-stiffness model is modified to a more generic scheme that includes the initial motor-to-load position offset. The cascaded design of the estimation algorithm, outlined in [9], is presented in more detail, and proofs of its convergence and stability properties in connection to the estimator's modular structure are provided. Moreover, a robustness analysis of the algorithm with respect 


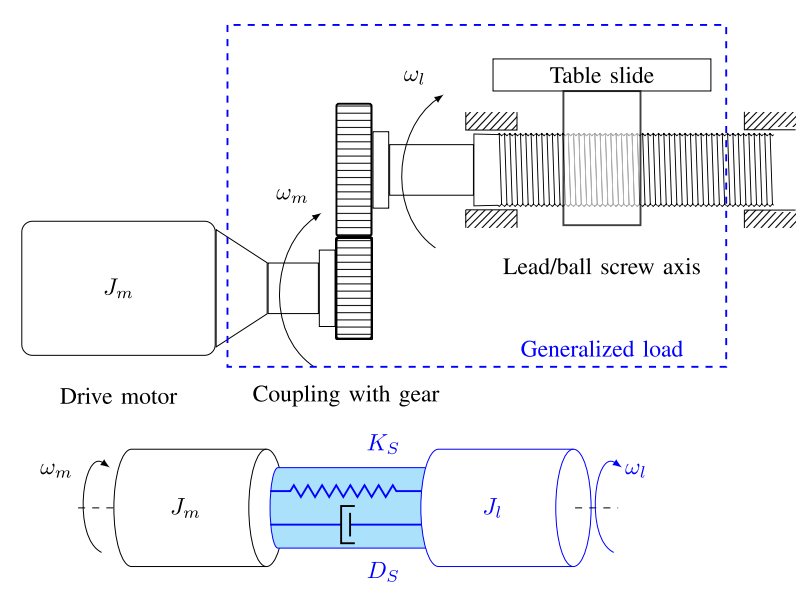

Fig. 1. Correspondence between mechanical drive-train and single-axis machine-tool systems. The angular velocities of the motor and the load are denoted by $\omega_{m}$ and $\omega_{l}$, respectively.

to modeling uncertainties is carried out and bounds are given for the deadzone estimation error. Finally, the theoretical findings are validated both with simulations and through a number of experiments performed on a real single-axis drive train with the state-of-the-art Siemens equipment.

This paper is organized as follows. Section II states the estimation problem discussed in this paper, describes the drivetrain system, and presents the modified backlash model based on varying shaft stiffness. Section III analytically describes the design of the deadzone estimation scheme and provides the convergence and stability proof for the adaptive estimator. The effect of model uncertainties on the performance of the algorithm is discussed in Section IV, where a proof for the boundedness of the estimation error is presented. Section V illustrates the estimator performance in a simulation environment. Experimental validation of the theoretical findings is provided in Section VI. Finally, conclusions are drawn and future work is discussed in Section VII.

\section{System Modeling ANd Problem Formulation}

\section{A. Drive-Train Modeling}

As discussed in [9], a typical single-axis machine tool can be described as a drive train, which consists of the drive motor, a flexible shaft with damping and backlash, and a generalized load with friction. The correspondence between the single-axis machine tool and the abstraction of the drive train can be seen in Fig. 1.

The drive component is a permanent magnet synchronous motor (PMSM) that is typically used for actuating linear axes in machine tools, especially for highly dynamic tasks. The motor is position-controlled with a cascade of a proportional and a proportional-integral controller, used for the position and the velocity loops, respectively.

In general, the electrical closed-loop dynamics is much faster than that of the mechanical system. Moreover, since the focus of this paper is on the identification of backlash, i.e., on the level of the accelerations, and since the torque produced by the motor is measured, the closed-loop electrical dynamics

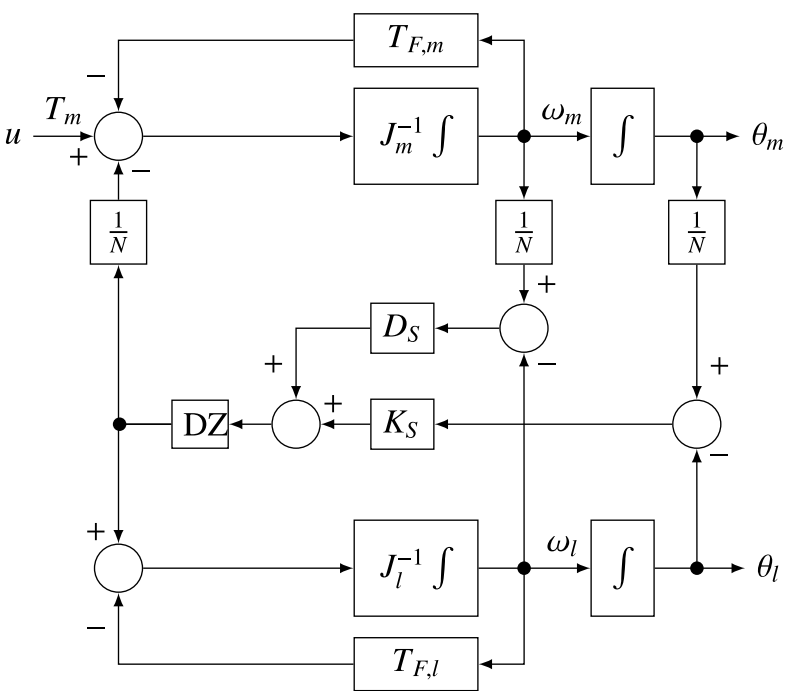

Fig. 2. Block diagram of the open-loop mechanical drive-train system. The block labeled "DZ" represents the backlash deadzone.

of the motor will not be considered in this paper. This does not affect the design of the estimation algorithms as it will become clearer later in the analysis.

The dynamics of the mechanical drive-train system reads

$$
\begin{aligned}
\dot{\omega}_{m} & =\frac{1}{J_{m}}\left(u-T_{F, m}\left(\omega_{m}\right)-\frac{1}{N} T_{l}^{\mathrm{BF}}(\boldsymbol{x})\right) \\
\dot{\theta}_{m} & =\omega_{m} \\
\dot{\omega}_{l} & =\frac{1}{J_{l}}\left(T_{l}^{\mathrm{BF}}(\boldsymbol{x})-T_{F, l}\left(\omega_{l}\right)\right) \\
\dot{\theta}_{l} & =\omega_{l}
\end{aligned}
$$

where $\omega_{m}, \theta_{m}$ and $\omega_{l}, \theta_{l}$ are the angular velocity and position of the motor and the load, respectively, $J_{m}$ and $J_{l}$ are the corresponding inertias and $N$ is the gearing ratio. In the backlash-free case, the interconnecting torque $T_{l}^{\mathrm{BF}}$ is given as

$$
T_{l}^{\mathrm{BF}}(\boldsymbol{x})=K_{S}\left(\frac{1}{N} \theta_{m}-\theta_{l}\right)+D_{S}\left(\frac{1}{N} \omega_{m}-\omega_{l}\right)
$$

where $K_{S}$ is the shaft stiffness and $D_{S}$ is the damping coefficient and the superscript "BF" stands for "backlash-free." The friction torques acting on the drive motor and the load express different frictional phenomena. $T_{F, m}$ comes mostly from the contacting surfaces of the motor bearings, while $T_{F, l}$ describes the total Coulomb and viscous friction in the load. The two friction torques are modeled as described in the following equations [10]:

$$
\begin{aligned}
T_{F, m}\left(\omega_{m}\right) & =T_{C, m} \operatorname{sgn}\left(\omega_{m}\right)+\beta_{m} \omega_{m} \\
T_{F, l}\left(\omega_{l}\right) & =T_{C, l} \operatorname{sgn}\left(\omega_{l}\right)+\beta_{l} \omega_{l}
\end{aligned}
$$

where $\operatorname{sgn}(\cdot)$ is the signum function defined in (63) in Appendix A. The parameters $\beta_{m}, \beta_{l}, T_{C, m}$, and $T_{C, l}$ are considered as constant. Fig. 2 shows the block diagram of the drive-train system in the open loop.

\section{B. Backlash Modeling}

Backlash shows up as a loss of engagement between two moving parts (e.g., motor and load) due to a developing gap 


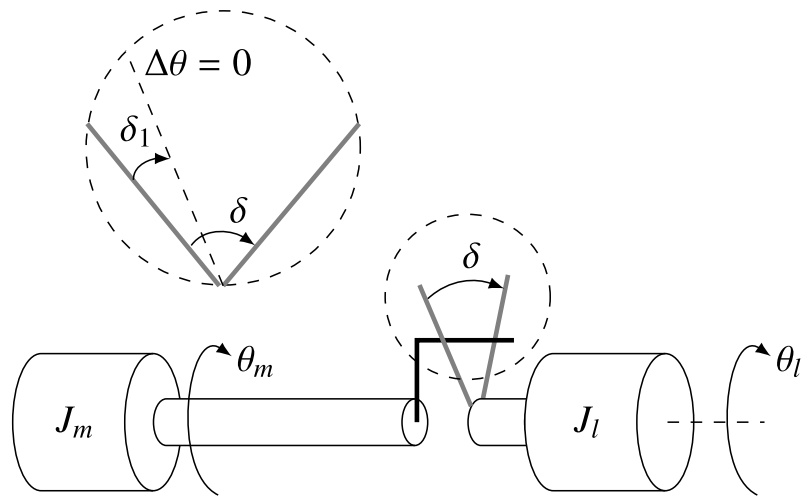

Fig. 3. Backlash illustration. The straight dashed line denotes the relative configuration between the motor and the load rotors that is taken as zero position difference. When the difference between the motor and the load position is larger than $-\delta_{1}$ or smaller than $\delta-\delta_{1}$, then the two shafts are disengaged and no torque is applied to the motor or the load.

(deadzone) in the coupling mechanisms, as shown in Fig. 3. The backlash phenomenon can be interpreted as a sudden change either in the load inertia or in the shaft stiffness. In general, the backlash torque is modeled after the restoring and damping torques in a mass-spring-damper system, while in the deadzone, these two torques are either vanishing or becoming very small, nearly zero. A number of studies have been carried out regarding the description of the torques due to backlash. The most intuitive and common one is the deadzone model [10], [11], where the interconnecting torque $T_{l}^{\mathrm{BF}}$ becomes zero inside the deadzone. Outside the deadzone, the angle difference is offset by the width of the deadzone angle. A similar approach is found in [12], where the ratio of the angle differences over the deadzone width is considered. A generic backlash operator based on a discontinuous inputoutput mapping was used in [13]-[15] to capture the hysteretic behavior of mechanical systems with gearing. Dynamical models described in [16] and [17] pertain to express the backlash torque as a sudden impact. The torque is again given by a mass-spring-damper system, where the elastic linear relative deformation of the two colliding coupling parts has its own stiff dynamics. Tao and Kokotovic [18] proposed a firstorder discontinuous model to describe the backlash between commanded and real input of dynamical systems. The inverse of the model is used for designing an adaptive control scheme. A different dynamical model is described in [11] and [19], where a backlash angle is defined and its dynamics is used for calculating the impact torque.

In [9], we introduced a smooth backlash model based on changing shaft stiffness to overcome the numerical difficulties present in the state-of-the-art discontinuous models. This model is modified in this paper to include an initial position offset between motor and load, as shown in Fig. 3.

Defining the angular position and velocity differences between drive motor and load as

$$
\begin{gathered}
\Delta \theta \triangleq \frac{1}{N} \theta_{m}-\theta_{l} \\
\Delta \omega \triangleq \frac{1}{N} \omega_{m}-\omega_{l}
\end{gathered}
$$

TABLE I

SySTEM MOdel NOMENCLATURE

\begin{tabular}{clc} 
Symbol & Description & Units \\
\hline \multicolumn{2}{l}{ States and Outputs } \\
\hline$\omega_{m}, \omega_{l} \quad$ Motor/load angular velocity & $\mathrm{rad} \mathrm{s}^{-1}$ \\
$\theta_{m}, \theta_{l}$ & Motor/load angular position & $\mathrm{rad}$ \\
\hline \multicolumn{2}{l}{ Inputs and disturbances } \\
\hline \multicolumn{2}{c}{ Torque command } \\
$T_{F, m}, T_{F, l} \quad$ Motor/load friction \\
$T_{l}^{B F}, T_{l}$ & Interconnecting/backlash torque & $\mathrm{N} \mathrm{m}$ \\
\hline Constant parameters & $\mathrm{N} \mathrm{m}$ \\
\hline$J_{m}, J_{l}$ & Motor/load inertia \\
$K_{S}$ & Shaft stiffness & $\mathrm{kg} \mathrm{m}^{2}$ \\
$D_{S}$ & Shaft damping coefficient & $\mathrm{N} \mathrm{m} \mathrm{rad}$ \\
$N$ & Gearing ratio & $\mathrm{N} \mathrm{m} \mathrm{s} \mathrm{rad}$ \\
& & - \\
$T_{C, m}, T_{C, l}$ & Coulomb friction on the motor/load & $\mathrm{N} \mathrm{m}_{m}$ \\
$\beta_{m}, \beta_{l}$ & Viscous friction coefficients & $\mathrm{N} \mathrm{m} \mathrm{s} \mathrm{rad}^{-1}$ \\
$\delta, \delta_{1}$ & Deadzone angle/position offset & $\operatorname{rad}^{2}$
\end{tabular}

the interconnecting torque $T_{l}^{\mathrm{BF}}$ in the deadzone model is replaced by the backlash torque

$$
T_{l}^{D Z}= \begin{cases}K_{S}\left(\Delta \theta+\delta_{1}\right)+D_{S} \Delta \omega, & \Delta \theta<-\delta_{1} \\ K_{S}\left(\Delta \theta+\delta_{1}-\delta\right)+D_{S} \Delta \omega, & \Delta \theta>\delta-\delta_{1} \\ 0, & 0 \leq \Delta \theta+\delta_{1} \leq \delta\end{cases}
$$

where $\delta$ is the width of the deadzone in rad. In the modified smooth model proposed in [9], the shaft stiffness and the backlash torque are described by

$$
\begin{aligned}
K_{\mathrm{BL}}= & \frac{K_{S}}{\pi}\left[\pi+\arctan \left(\alpha\left(\Delta \theta-\delta+\delta_{1}\right)\right)\right. \\
T_{l}(\boldsymbol{x}, \delta)= & {\left[\Delta \theta+\delta_{1}-\frac{\delta}{2} \cdot\left(1+\arctan \left(\alpha\left(\Delta \theta+\delta_{1}\right)\right)\right]\right.} \\
& \left.\left.\cdot K_{\mathrm{BL}}(\Delta \theta, \delta)\right)+\frac{D_{S}}{K_{S}} \Delta \omega\right]
\end{aligned}
$$

where

$$
\boldsymbol{x} \triangleq\left[\begin{array}{cccc}
\omega_{m} & \theta_{m} & \omega_{l} & \theta_{l}
\end{array}\right]^{T}
$$

is the state vector, $0 \leq \delta_{1} \leq \delta$ is the initial motor-load position offset, and $\alpha$ is a large positive real number that parameterizes how steep the change in the stiffness is. For $\alpha \rightarrow \infty$, it is clear that $T_{l} \rightarrow T_{l}^{D Z}$. This can also be seen in Fig. 4, where the stiffness $K_{\mathrm{BL}}(\Delta \theta, \delta)$ and the corresponding backlash torques are plotted for different values of the parameter $\alpha$. A complete explanation of the most important variables and notation used in the modeling of the system is provided in Table I.

\section{Problem Formulation}

The collective objective can be summarized in the following problem formulation. 

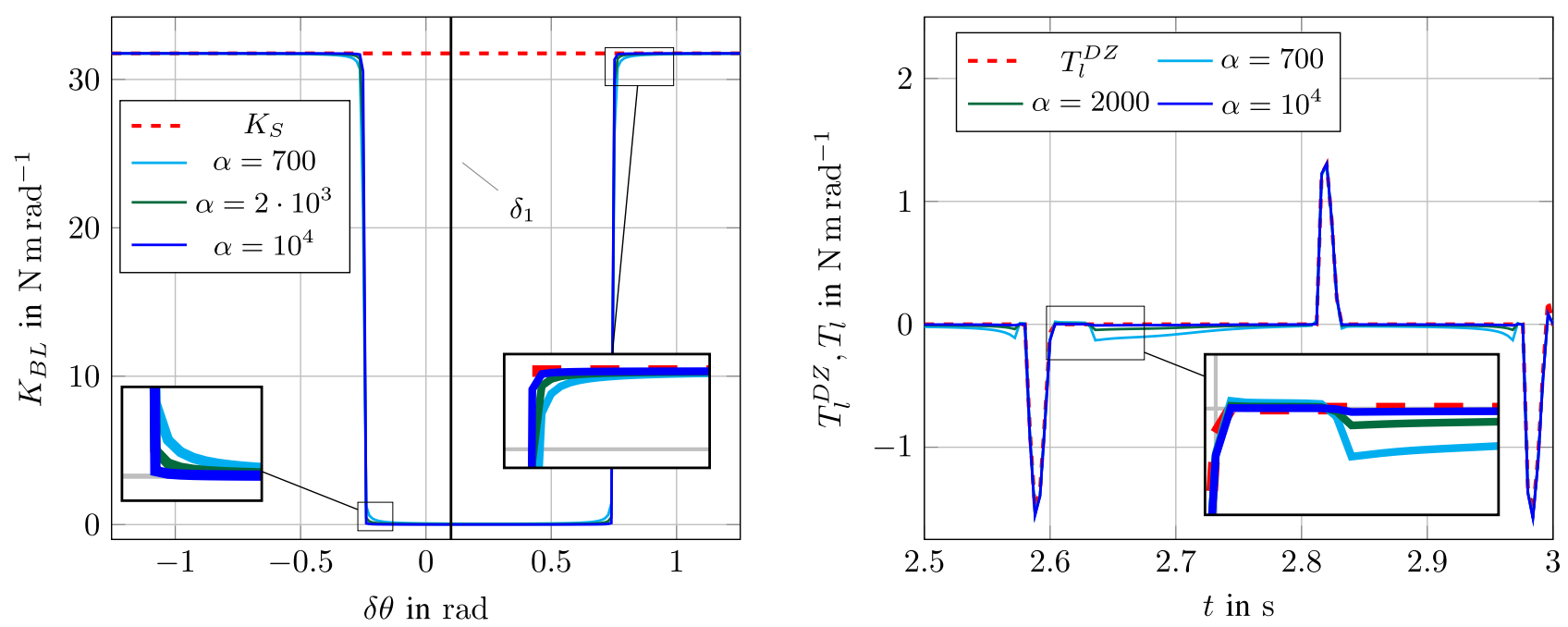

Fig. 4. Left: shaft stiffness varying between two values. The larger the value of $\alpha$ is, the steeper the change in the stiffness is. Right: backlash torque for a sinusoidal motion profile using the deadzone model (red dotted curve) and the varying-stiffness model (solid lines) for different values of $\alpha$.

Problem 1 (Deadzone Angle Estimation): Consider the single-axis drive-train system described in (1)-(4), (6), and (7), where $T_{l}^{\mathrm{BF}}$ is replaced by the backlash torque defined in (11) and (12) with the known initial position offset $\delta_{1}$. Design an online dynamic estimator for the deadzone angle $\delta$, such that the estimate $\hat{\delta}$ fulfills the following requirements.

1) Convergence to a compact set containing the real deadzone angle value $\delta=\delta^{*}$.

2) Maximum steady-state absolute estimation error is less than $10^{-2}$ rad.

\section{BACKLASH DEADZONE Angle Estimation}

The estimation of the deazone angle belongs to the family of problems of online parameter estimation in systems with nonlinear parameterization, treated in numerous works in the literature. The reader is indicatively referred to [20]-[25].

The approach presented in [9] is partially based on a method for parameter estimation in nonlinearly parameterized systems presented in [26]. The basic idea relates to estimating a perturbation of the system dynamics that depends on the unknown parameter and then finding an adaptation law for estimating the parameter itself. In [26], both the perturbation and the unknown parameter are estimated simultaneously using the two estimation blocks (perturbation and parameter) in the feedback interconnection. In [9], these two estimation tasks are separated to allow for an independent design for each of them. In this section, the estimation scheme presented in [9] is elaborated and a proof of its convergence and stability properties is provided.

\section{A. Method Overview}

As in [9], the dynamics of the load shown in (3) is used to apply the method for the deadzone angle estimation. This is because all the states are measured and the unknown parameter $\delta$ affects the dynamics of both the motor and the load in the same way, i.e., through the torque $T_{l}$. The load velocity subsystem can be rewritten in the form

$$
\dot{\omega}_{l}=-\frac{1}{J_{l}} T_{F, l}\left(\omega_{l}\right)+\phi(x, \delta)
$$

with

$$
\phi(x, \delta) \triangleq \frac{1}{J_{l}} T_{l}(x, \delta)
$$

and $\delta=\delta^{*}$ being the unknown parameter, which belongs to a compact set $\mathcal{D} \subset \mathbb{R}_{\geq 0}$. The method is divided in two parts.

1) Obtain an estimation $\hat{\phi}$ of the perturbation $\phi\left(x, \delta^{*}\right)$.

2) Derive an adaptation law for the deadzone angle

$$
\dot{\hat{\delta}} \triangleq \rho(\boldsymbol{x}, \hat{\phi}, \hat{\delta})
$$

based on $\hat{\phi}$.

\section{B. Sliding-Mode Perturbation Observer}

A second-order SMO is used for finding an estimate of $\phi$. Its structure is given by [27], [28]

$$
\dot{\hat{\omega}}_{l}=-\frac{1}{J_{l}} T_{F, l}\left(\omega_{l}\right)+v
$$

where $\tilde{\omega}_{l}=\omega_{l}-\hat{\omega}_{l}$ is the velocity estimation error and $v$ is an appropriate high frequency term, called the injection signal, that depends on the innovation signal $\tilde{\omega}_{l}$.

From (3) and (16), the dynamics of the velocity estimation error in the absence of any model or parameters uncertainties reads

$$
\dot{\tilde{\omega}}_{l}=\dot{\omega}_{l}-\dot{\hat{\omega}}_{l}=\frac{1}{J_{l}} T_{l}\left(\boldsymbol{x}, \delta^{*}\right)-v .
$$

With the sliding manifold that is defined as

$$
S \triangleq\left\{\tilde{\omega}_{l} \in \mathbb{R}: \tilde{\omega}_{l}=\dot{\tilde{\omega}}_{l}=0\right\}
$$

the design of $v$ is given in [29]

$$
v=k_{1}\left|\tilde{\omega}_{l}\right|^{\frac{1}{2}} \operatorname{sgn}\left(\tilde{\omega}_{l}\right)+k_{2} \int_{0}^{t} \operatorname{sgn}\left(\tilde{\omega}_{l}(\tau)\right) d \tau
$$


where $k_{1}$ and $k_{2}$ are positive gains. The resulting observer is called the supertwisting sliding-mode observer (STSMO), and it is proven in [30] and [31] that for appropriate positive gains $k_{1}$ and $k_{2}$, the injection signal $v$ brings the observer error dynamics on the sliding manifold $S$ in finite time, where it remains thereafter.

If the error dynamics reaches the sliding manifold, then $\dot{\tilde{\omega}}_{l}=\tilde{\omega}_{l}=0$ for all future times, which from (17) leads to

$$
v=\frac{1}{J_{l}} T_{l}\left(\boldsymbol{x}, \delta^{*}\right) .
$$

In other words, if the injection signal $v$ is designed such that the estimation error dynamics reaches the sliding manifold $S$ and remains thereafter, then the unknown perturbation $\phi=\left(1 / J_{l}\right) T_{l}$ is indirectly calculated from (20) [32]. Hence, the unknown perturbation can be estimated at each time instant $t$ by

$$
\hat{\phi}=k_{1}\left|\tilde{\omega}_{l}\right|^{\frac{1}{2}} \operatorname{sgn}\left(\tilde{\omega}_{l}\right)+k_{2} \int_{0}^{t} \operatorname{sgn}\left(\tilde{\omega}_{l}(\tau)\right) d \tau .
$$

Remark 1: The choice of a second-order SMO for a system of relative degree 1 (the subsystem is scalar) was made due to the property of higher order SMOs of alleviating the chattering in the injection and estimation signals [28], [33].

Remark 2: Apart from the appropriate selection of $k_{1}$ and $k_{2}$, the finite-time estimation additionally requires that $T_{l}$ and $\dot{T}_{l}$ are bounded. This is ensured by the boundedness of the state vector and the smoothness of the backlash model. However, the bound on $\dot{T}_{l}$ is proportional to $\alpha$. This means that the closer the model is to the deadzone model, the larger this bound will be, which in turn leads to higher gains for the observer and, consequently, more chattering in the estimation signal.

Remark 3: Since $\left|\dot{T}_{l}\right|$ is bounded by a positive number $\rho$, selecting the STSMO gains $k_{1}$ and $k_{2}$, such that $k_{2}>\rho, k_{1}>$ $1.41\left(k_{2}+\rho\right)^{1 / 2}$ ensures finite-time convergence of the velocity estimation error to the sliding manifold [34]. Given the discontinuous nature of the backlash phenomenon (abrupt engagement/disengagement between motor and load), such a selection may lead to large gains and unacceptable levels of chatter in the estimation signal. An alternative way to tune the STSMO is to start with small values of $k_{1}$ and $k_{2}$ and increase $k_{1}$ until before substantial chatter shows up in the estimation signal. At that point, the system will not be in the sliding motion, which means that $\dot{\tilde{\omega}}_{l}(t)=s\left(t, \omega_{l}(t)\right) \Rightarrow v=$ $\left(1 / J_{l}\right) T_{l}+s\left(t, \omega_{l}(t)\right)$, where $s\left(t, \omega_{l}(t)\right) \neq 0$ is bounded for bounded $\omega_{l}$. Further increase of $k_{2}$ will improve the accuracy of the perturbation estimation without significantly increasing the chatter. The effect of not perfect estimation of $\phi$ on the deadzone angle estimation accuracy is extensively discussed in Section IV.

\section{Adaptive Backlash Angle Estimator}

The adaptive deadzone angle estimator is based on the design proposed in [26] for the estimation of unknown parameters in nonlinearly parameterized systems. For the rest of the analysis, we consider that the unknown parameter $\delta=\delta^{*}$ lies in a compact set $\mathcal{D}=\left[0, \delta_{\max }\right] \subset \mathbb{R}_{\geq 0}$, where $\delta_{\max }$ is the largest considered deadzone angle, and we define the backlash angle estimation error as

$$
\tilde{\delta}=\delta^{*}-\hat{\delta} .
$$

The following assumptions are made.

Assumption 1: The deadzone angle is constant or slowly varying, i.e.,

$$
\dot{\delta}^{*} \approx 0 .
$$

Two auxiliary functions, $\sigma: \mathbb{R}^{4} \rightarrow \mathbb{R}_{\geq 0}$ and $\mu: \mathbb{R}^{4} \times \mathcal{D} \rightarrow \mathbb{R}$, are needed for proving convergence and stability. Both are bounded for bounded state vector $\boldsymbol{x}$ and piecewise continuous. They have the following properties.

Property 1: For all pairs, $\delta_{A}, \delta_{B} \in \mathcal{D}$

$$
\mu\left(\boldsymbol{x}, \delta_{A}\right) \frac{1}{J_{l}} \frac{\partial T_{l}}{\partial \delta}\left(\boldsymbol{x}, \delta_{B}\right) \geq \sigma(\boldsymbol{x}) .
$$

Property 2: There exists a positive real constant number $L>0$ such that $\forall \delta_{A}, \delta_{B} \in \mathcal{D}$

$$
\frac{1}{J_{l}}\left|T_{l}\left(\boldsymbol{x}, \delta_{B}\right)-T_{l}\left(\boldsymbol{x}, \delta_{A}\right)\right| \leq L \sqrt{\sigma(\boldsymbol{x})}\left|\delta_{B}-\delta_{A}\right| .
$$

The selection of functions $\mu(\boldsymbol{x}, \delta)$ and $\sigma(\boldsymbol{x})$ will be detailed later in this section. A persistence of excitation (PE) condition is also needed.

Assumption 2: There exist positive real numbers $T$ and $\varepsilon$ such that $\forall t \in \mathbb{R}_{\geq 0}$

$$
\int_{t}^{t+T} \sigma(x(\tau)) d \tau \geq \varepsilon .
$$

The main result of this paper is an adaptive estimator design that is expressed in Theorem 1.

Theorem 1: Consider the dynamics of the load velocity given by (11)-(14) and the functions $\mu(\boldsymbol{x}, \delta)$ and $\sigma(x)$ with properties 1 and 2. Also, consider an estimate $\hat{\phi}$ of the interconnecting perturbation $\phi=\phi\left(\boldsymbol{x}, \delta^{*}\right)$ with the associated estimation error defined as

$$
\tilde{\phi} \triangleq \phi-\hat{\phi} .
$$

The adaptive estimator

$$
\dot{\hat{\delta}}=\rho(\boldsymbol{x}, \hat{\phi}, \hat{\delta})=\operatorname{Proj}\left[\hat{\delta}, \gamma \mu(\boldsymbol{x}, \hat{\delta})\left(\hat{\phi}-\frac{1}{J_{l}} T_{l}(\boldsymbol{x}, \hat{\delta})\right)\right]
$$

with $\gamma>0$ the adaptation gain and $\operatorname{Proj}(\cdot, \cdot)$ the projection operator defined in Appendix B, ensures that the estimation error $\tilde{\delta}$ asymptotically converges to 0 , uniformly in $\boldsymbol{x}$ if assumptions 1 and 2 are satisfied and $\tilde{\phi}^{*}=0$ is an uniformly globally asymptotically stable (UGAS) equilibrium of the perturbation estimation error dynamics $\tilde{\phi}$.

Proof: The proof is inspired by the proof of [26, Proposition 4] and the stability theory of interconnected systems in [35]. Under Assumption 1 and the definition of the perturbation estimation error in (25), the dynamics of the parameter estimation error $\tilde{\delta}$ is written as

$$
\dot{\tilde{\delta}}=-\operatorname{Proj}\left[\hat{\delta}, \gamma \mu(\boldsymbol{x}, \hat{\delta})\left(\phi-\tilde{\phi}-\frac{1}{J_{l}} T_{l}(\boldsymbol{x}, \hat{\delta})\right)\right] .
$$




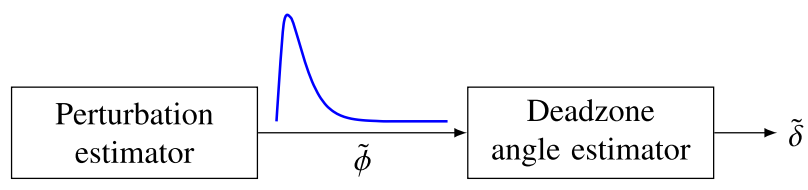

Fig. 5. Cascaded interconnection of the perturbation and angle estimation error systems.

From (27), it can be easily seen that the total error dynamics can be described as the cascaded interconnection of the unperturbed error system given by (27) for $\tilde{\phi} \equiv 0$ and the output $\tilde{\phi}$ of the (interconnecting torque) perturbation estimation error system, as shown in Fig. 5.

The proof is now split in two parts. First, it is shown that the unperturbed system of the deadzone angle estimation error is uniformly locally exponentially stable (ULES), and then, it will be shown that a UGAS estimate of $\phi$ renders the total cascaded system UGAS.

For $\tilde{\phi}=0$ in (27), the unperturbed error dynamics reads

$$
\dot{\tilde{\delta}}=-\operatorname{Proj}\left[\hat{\delta}, \gamma \mu(\boldsymbol{x}, \hat{\delta}) \frac{1}{J_{l}}\left(T_{l}\left(\boldsymbol{x}, \delta^{*}\right)-T_{l}(\boldsymbol{x}, \hat{\delta})\right)\right] .
$$

The shortened notation

$$
\begin{aligned}
& \mu \triangleq \mu(\boldsymbol{x}, \hat{\delta}) \\
& T_{l} \triangleq T_{l}\left(\boldsymbol{x}, \delta^{*}\right) \\
& \hat{T}_{l} \triangleq T_{l}(\boldsymbol{x}, \hat{\delta}) .
\end{aligned}
$$

will be used in the following for brevity. Define the Lyapunov function candidate

$$
V(t, \tilde{\delta})=\frac{1}{2}\left(\frac{1}{\gamma}-\kappa \int_{t}^{\infty} e^{(t-\tau)} \sigma(\boldsymbol{x}(\tau)) d \tau\right) \tilde{\delta}^{2}
$$

where $\kappa$ is a real positive number to be defined. The function is positive definite since

$$
\alpha_{1}(|\tilde{\delta}|) \leq V(t, \tilde{\delta}) \leq \alpha_{2}(|\tilde{\delta}|)
$$

with $\alpha_{1}$ and $\alpha_{2}$ class- $\mathcal{K}_{\infty}$ functions (see Definition 3 ) defined as

$$
\begin{aligned}
& \alpha_{1}(|\tilde{\delta}|)=\left(\frac{1}{\gamma}-\kappa \sup _{x \in \mathcal{B}_{r}} \sigma(\boldsymbol{x})\right)|\tilde{\delta}|^{2} \\
& \alpha_{2}(|\tilde{\delta}|)=\frac{1}{\gamma}|\tilde{\delta}|^{2}
\end{aligned}
$$

for $\kappa<1 /\left(\gamma \sup _{x \in \mathcal{B}_{r}} \sigma(x)\right)$, where $\mathcal{B}_{r}=\left\{\boldsymbol{x} \in \mathbb{R}^{4} \mid\|x\| \leq\right.$ $r, r<\infty\}$. The time derivative of $V$ along the trajectories of the estimation error reads

$$
\begin{aligned}
\dot{V}(t, \tilde{\delta}) & \frac{\partial V}{\partial t}(t, \tilde{\delta})+\frac{\partial V}{\partial \tilde{\delta}}(t, \tilde{\delta}) \dot{\tilde{\delta}} \\
= & \frac{1}{2} \tilde{\delta}^{2} \frac{\partial}{\partial t}\left[-\kappa \int_{t}^{\infty} e^{(t-\tau)} \sigma(\boldsymbol{x}(\tau)) d \tau\right] \\
& +\tilde{\delta}\left(\frac{1}{\gamma}-\kappa \int_{t}^{\infty} e^{(t-\tau)} \sigma(\boldsymbol{x}(\tau)) d \tau\right) \dot{\tilde{\delta}} \\
= & \frac{\kappa}{2} \tilde{\delta}^{2}\left[\frac{\partial}{\partial t} \int_{0}^{t} e^{(t-\tau)} \sigma(\boldsymbol{x}(\tau)) d \tau-\int_{0}^{\infty} \frac{\partial}{\partial t} e^{(t-\tau)} \sigma(\boldsymbol{x}(\tau)) d \tau\right] \\
& -\tilde{\delta}\left(\frac{1}{\gamma}-\kappa \int_{t}^{\infty} e^{(t-\tau)} \sigma(\boldsymbol{x}(\tau)) d \tau\right) \operatorname{Proj}\left[\hat{\delta}, \gamma \mu \cdot \frac{T_{l}-\hat{T}_{l}}{J_{l}}\right] .
\end{aligned}
$$

Using the property

$$
-\tilde{\delta} \frac{1}{\gamma} \operatorname{Proj}\left[\hat{\delta}, \gamma \mu \cdot \frac{1}{J_{l}}\left(T_{l}-\hat{T}_{l}\right)\right] \leq-\tilde{\delta} \mu \cdot \frac{1}{J_{l}}\left(T_{l}-\hat{T}_{l}\right)
$$

presented in [36, Lemma E.1], as well as, the property

$$
\int_{0}^{\infty} e^{(t-\tau)} \sigma(x(\tau)) d \tau \geq e^{-T} \int_{t}^{t+T} \sigma(x(\tau)) d \tau, \quad T>0
$$

(see Appendix D for a proof), (32) gives

$$
\begin{aligned}
\dot{V}(t, \tilde{\delta}) \leq & \frac{\kappa}{2} \tilde{\delta}^{2}\left(\sigma(\boldsymbol{x})-e^{-T} \int_{t}^{t+T} \sigma(\boldsymbol{x}(\tau)) d \tau\right) \\
& -\tilde{\delta}\left(\frac{1}{\gamma}-\kappa \int_{t}^{\infty} e^{(t-\tau)} \sigma(\boldsymbol{x}(\tau)) d \tau\right) \gamma \mu \cdot \frac{T_{l}-\hat{T}_{l}}{J_{l}} \\
\leq & \frac{\kappa}{2} \tilde{\delta}^{2}\left(\sigma(\boldsymbol{x})-e^{-T} \int_{t}^{t+T} \sigma(\boldsymbol{x}(\tau)) d \tau\right)-\tilde{\delta} \mu \frac{T_{l}-\hat{T}_{l}}{J_{l}} \\
& +|\tilde{\delta}| \kappa \gamma\left|\int_{t}^{\infty} e^{(t-\tau)} \sigma(\boldsymbol{x}(\tau)) d \tau\right||\mu| \frac{1}{J_{l}}\left|T_{l}-\hat{T}_{l}\right| \\
= & \frac{\kappa}{2} \tilde{\delta}^{2}\left(\sigma(\boldsymbol{x})-e^{-T} \int_{t}^{t+T} \sigma(\boldsymbol{x}(\tau)) d \tau\right) \\
& -\tilde{\delta} \int_{0}^{1} \mu(\boldsymbol{x}, \hat{\delta}) \cdot \frac{1}{J_{l}} \frac{\partial T_{l}}{\partial \delta}(\boldsymbol{x}, \hat{\delta}+p \tilde{\delta}) \tilde{\delta} d p \\
& +|\tilde{\delta}| \kappa \gamma\left|\int_{t}^{\infty} e^{(t-\tau)} \sigma(\boldsymbol{x}(\tau)) d \tau\right||\mu| \frac{1}{J_{l}}\left|T_{l}-\hat{T}_{l}\right|
\end{aligned}
$$

where [37, Th. 11.1] (see Appendix C) was used. Using Assumptions 1 and 2 and Properties 1 and 2 in the inequality mentioned earlier and introducing $M$ and $\Sigma$ as upper bounds for $|\mu(\boldsymbol{x}, \delta)|$ and $|\sigma(\boldsymbol{x})|$, respectively, lead to

$$
\begin{aligned}
\dot{V} \leq-\left(1-\frac{\kappa}{2}\right) \sigma(\boldsymbol{x}) \tilde{\delta}^{2}-\frac{\kappa}{2} \varepsilon e^{-T} \tilde{\delta}^{2} & \\
& +\kappa \gamma \cdot M \cdot \Sigma \cdot L \sqrt{\sigma(\boldsymbol{x})}|\tilde{\delta}|^{2}=-\boldsymbol{\psi}^{T} Q \boldsymbol{\psi}
\end{aligned}
$$

where

$$
\begin{aligned}
\boldsymbol{\psi}^{T} & =[|\tilde{\delta}| \sqrt{\sigma(\boldsymbol{x})}|\tilde{\delta}|] \\
Q & =\left[\begin{array}{cc}
\frac{\kappa}{2} \varepsilon e^{-T} & -\frac{\kappa}{2} \gamma \cdot M \cdot \Sigma \cdot L \\
-\frac{\kappa}{2} \gamma \cdot M \cdot \Sigma \cdot L & 1-\frac{\kappa}{2}
\end{array}\right]
\end{aligned}
$$

and the property

$$
\int_{t}^{\infty} e^{(t-\tau)} d \tau=1
$$

was used. From inequality (36), it can be seen that $\dot{V}$ is negative definite if $\kappa$ is chosen as

$$
\kappa<\frac{2}{1+\frac{e^{T}}{\varepsilon} M^{2} \Sigma^{2} \gamma^{2} L^{2}} .
$$

For such $\kappa$ and by defining

$$
\lambda_{\min }(Q)=\min _{i \in\{1,2\}}\left\{\lambda_{i} \in \mathbb{R}_{\geq 0} \mid P_{Q}\left(\lambda_{i}\right)=0\right\}
$$

where $P_{Q}$ is the characteristic polynomial of $Q,(36)$ is written

$$
\dot{V}(t, \tilde{\delta}) \leq-\underbrace{\lambda_{\min }(Q)}_{\alpha_{3}}\left\|\frac{1}{\sigma(\boldsymbol{x})}\right\|_{2}^{2}|\tilde{\delta}|^{2} \leq-\alpha_{3}|\tilde{\delta}|^{2} .
$$


Moreover,

$$
\begin{aligned}
\left|\frac{\partial V}{\partial \tilde{\delta}}\right| & =\left|\tilde{\delta}\left(\frac{1}{\gamma}-\kappa \int_{t}^{\infty} e^{(t-\tau)} \sigma(\boldsymbol{x}(\tau)) d \tau\right)\right| \\
& \leq\left|\frac{1}{\gamma}-\kappa e^{-T} \int_{t}^{t+T} \sigma(\boldsymbol{x}(\tau)) d \tau\right||\tilde{\delta}| \\
& \leq\left|\frac{1}{\gamma}-\kappa e^{-T} \Sigma \int_{t}^{t+T} d \tau\right||\tilde{\delta}| \\
& \leq \underbrace{\left|\frac{1}{\gamma}-\kappa e^{-T} \Sigma T\right|}_{\alpha_{4}}|\tilde{\delta}|=\alpha_{4}|\tilde{\delta}|
\end{aligned}
$$

which implies that $(\partial V / \partial \tilde{\delta})$ is uniformly bounded (UB). Then, the equilibrium point $\tilde{\delta}^{*}=0$ is ULES [38].

Next, it will be shown that the system in (27) is input-tostate stable (ISS) (see Definition 5) with respect to $\tilde{\phi}$.

Taking the time derivative of $V$ along the trajectories of the perturbed system (27) now and following the same steps as for the unperturbed system give:

$$
\begin{aligned}
\dot{V}(t, \tilde{\delta}) & \\
\leq & -\alpha_{3}|\tilde{\delta}|^{2}-\tilde{\delta}\left(\frac{1}{\gamma}-\kappa \int_{t}^{\infty} e^{t-\tau} \sigma(\boldsymbol{x}(\tau)) d \tau\right) \gamma \mu \tilde{\phi} \\
\leq & -\alpha_{3}|\tilde{\delta}|^{2}+|\tilde{\delta}|\left(1-\kappa \gamma e^{-T} \int_{t}^{t+T} \sigma(\boldsymbol{x}(\tau)) d \tau\right) \mu|\tilde{\phi}| \\
\leq & -\alpha_{3}|\tilde{\delta}|^{2}+|\tilde{\delta}|\left(1-\kappa \gamma e^{-T} \varsigma \cdot T\right) M|\tilde{\phi}| \\
\leq & |\tilde{\delta}|\left[\left(1-\kappa \gamma e^{-T} \varsigma \cdot T\right) M|\tilde{\phi}|-\vartheta \alpha_{3}|\tilde{\delta}|\right] \\
& -(1-\vartheta) \alpha_{3}|\tilde{\delta}|^{2} \leq-(1-\vartheta) \alpha_{3}|\tilde{\delta}|^{2}
\end{aligned}
$$

for all $\tilde{\delta} \in \mathbb{R}$ that satisfy

$$
|\tilde{\delta}| \geq \frac{\left(1-\kappa \gamma e^{-T} \varsigma \cdot T\right) M}{\alpha_{3} \vartheta}|\tilde{\phi}|=\underbrace{R|\tilde{\phi}|}_{r(|\tilde{\phi}|)}
$$

with $0<\vartheta<1$

$$
R \triangleq \frac{\left(1-\kappa \gamma e^{-T} \varsigma \cdot T\right) M}{\alpha_{3} \vartheta}
$$

and $\varsigma \geq 0$ being a lower bound for $|\sigma(\boldsymbol{x})|$. Then, according to [38, Th. 419], the perturbed system (27) is ISS with respect to $\tilde{\phi}$.

Last, since the unperturbed system (28) is ULES and the perturbed system (27) is ISS with respect to $\tilde{\phi}$, then if $\tilde{\phi}^{*}=0$ is UGAS, then the deadzone angle estimation error $\tilde{\delta}$ asymptotically converges to the real value $\delta^{*}[35$, Lemma 1], which completes the proof.

The uniform global asymptotic stability of the equilibrium $\tilde{\phi}^{*}=0$ is theoretically guaranteed by the STSMO, which ensures finite-time stabilization of the perturbation estimation error to the origin (which is a stronger stability property).

The final steps of the design include determining $\mu(\boldsymbol{x}, \delta)$ and $\sigma(\boldsymbol{x})$ to satisfy Properties 1 and 2. Selecting $\mu(\boldsymbol{x}, \delta)$ as

$$
\mu(\boldsymbol{x}, \delta)=\frac{J_{l}}{K_{S}^{2}} \frac{\partial T_{l}}{\partial \delta}(\boldsymbol{x}, \delta)
$$

makes condition (22) satisfied, with

$$
\sigma(\boldsymbol{x})=\frac{1}{K_{S}^{2}}\left(\left.\frac{\partial T_{l}}{\partial \delta}\right|_{\delta=\Delta}\right)^{2}=\frac{1}{\pi^{2}}\left[\chi_{1}(\boldsymbol{x}, \Delta)+\chi_{2}(\boldsymbol{x}, \Delta)\right]^{2}
$$

where the functions $\chi_{1}, \chi_{2}$ are defined as

$$
\begin{aligned}
\chi_{1}(\boldsymbol{x}, \delta)= & \frac{1}{2}(1+\operatorname{sgn}(\Delta \theta)) \\
& \cdot\left[\pi+\arctan \left(\alpha\left(\Delta \theta-\delta+\delta_{1}\right)\right)-\arctan \left(\alpha\left(\Delta \theta+\delta_{1}\right)\right)\right] \\
\chi_{2}(\boldsymbol{x}, \delta)= & {\left[\Delta \theta+\delta_{1}-\frac{\delta}{2} \cdot(1+\operatorname{sgn}(\Delta \theta))+\frac{D_{S}}{K_{S}} \Delta \omega\right] } \\
& \cdot \frac{\alpha}{1+\left[\alpha\left(\Delta \theta+\delta_{1}-\delta\right)\right]^{2}}
\end{aligned}
$$

and $\Delta$ is a large finite positive value with $\Delta>\delta_{\max }$.

Since $\boldsymbol{x}$ and $\sigma(\boldsymbol{x})$ are bounded and $\mathcal{D}$ is compact, it is easy to show that there exits $L>0$, such that condition (23) holds. Indeed

$$
\left|\frac{\partial T_{l}}{\partial \delta}\right|=\left|\frac{K_{S}}{\pi}\left[\chi_{1}(\boldsymbol{x}, \delta)+\chi_{2}(\boldsymbol{x}, \delta)\right]\right|
$$

is bounded, since $\boldsymbol{x}$ is bounded and $\delta$ belongs to a compact set. Assume that $\sigma(\boldsymbol{x}) \neq 0$ (for large $\Delta$, the function $\sigma$ approaches 0 asymptotically) and let $\varsigma>0$ be a lower bound for $\sigma(\boldsymbol{x})$. Equation (48) implies that there exists positive constant real number $L_{0}$, such that for each pair $\left(\delta^{*}, \hat{\delta}\right)$, the following inequality holds:

$$
\begin{aligned}
\frac{1}{J_{l}}\left|T_{l}\left(\boldsymbol{x}, \delta^{*}\right)-T_{l}(\boldsymbol{x}, \hat{\delta})\right| & \leq L_{0}|\tilde{\delta}| \leq \underbrace{\frac{L_{0}}{\sqrt{\varsigma}}}_{L} \sqrt{\sigma(\boldsymbol{x})}|\tilde{\delta}| \\
& =L \sqrt{\sigma(\boldsymbol{x})}|\tilde{\delta}|
\end{aligned}
$$

which shows that Property 2 is obtained.

Equations (45)-(47) show that the PE condition (24) does not hold if during the time interval $[t, t+T]$, the system is always within the deadzone. This, however, is expected, since in that case, there is no engagement between motor and load and, hence, no information about the stiffness of the shaft connecting them.

Remark 4: The selection of functions $\mu(x, \delta), \sigma(x)$ is not unique. The specific choice of $\mu(x, \hat{\delta})$ in (44) results into a gradient-type adaptive law which is very common in the literature of adaptive techniques. This choice, although it is sufficient in the specific estimation problem, cannot be generalized for nonlinearly parameterized systems, since it does not always guarantee parameter convergence.

The adaptive law for the parameter estimate $\hat{\delta}$ is finally given by

$$
\dot{\hat{\delta}}=\operatorname{Proj}\left\{\hat{\delta}, \gamma \frac{J_{l}}{K_{S}^{2}} \frac{\partial T_{l}}{\partial \delta}(\boldsymbol{x}, \hat{\delta})\left[\hat{\phi}-\frac{1}{J_{l}} T_{l}(\boldsymbol{x}, \hat{\delta})\right]\right\}, \quad \gamma>0
$$

where

$$
\frac{\partial T_{l}}{\partial \delta}(\boldsymbol{x}, \hat{\delta})=-\frac{K_{S}}{\pi}\left[\chi_{1}(\boldsymbol{x}, \hat{\delta})+\chi_{2}(\boldsymbol{x}, \hat{\delta})\right]
$$

The complete estimator design is shown in Fig. 6 and is summarized in the Algorithm 1. 


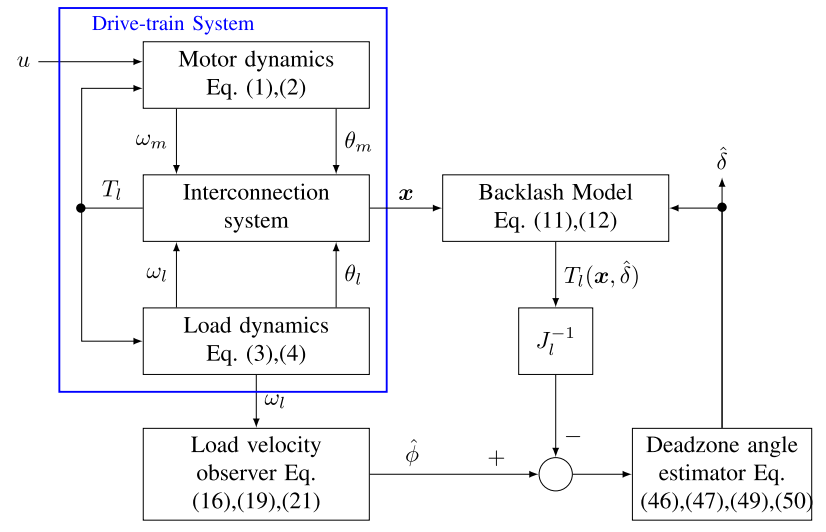

Fig. 6. Block diagram of the single-axis mechanical drive-train system and the estimation scheme.

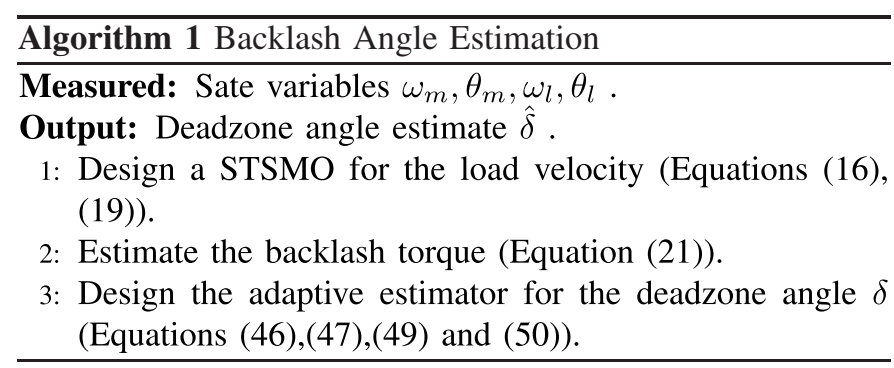

\section{Robustness Analysis}

Section III was discussed the problem of estimating the deadzone angle in the case where an asymptotic estimate or an exact measurement of the interconnecting torque $T_{l}=J_{l} \phi$ is available. Specifically, it was shown that the adaptive estimator ensures that the equilibrium point $\tilde{\delta}^{*}=0$ of the estimation error system is ULES if an exact measurement of $\phi$ is available or UGAS if an asymptotic estimate $\hat{\phi}$ of the real perturbation $\phi$ is used instead.

In real applications, however, such assumptions on the availability of a measurement or even an asymptotic estimate of $\phi$ may not necessarily hold. The effect of measurement noise, parameters' uncertainty, and modeling mismatches allow, under the assumption of boundedness of the uncertainties, at best for convergence of the interconnecting perturbation estimation error to a compact set.

In order to illustrate this better, consider the real backlash torque $T_{l}^{\prime}$ defined as

$$
\begin{aligned}
T_{l}^{\prime}= & {\left[\Delta \theta+\delta_{1}-\frac{\delta^{*}}{2} \cdot(1+\operatorname{sgn}(\Delta \theta))+\frac{D_{S}+\Delta D_{S}}{K_{S}+\Delta K_{S}} \Delta \omega\right] } \\
& \cdot K_{\mathrm{BL}}^{\prime}\left(\Delta \theta, \delta^{*}\right)+\Delta_{m}(t) \\
K_{\mathrm{BL}}^{\prime}= & \frac{K_{S}+\Delta K_{S}}{\pi} \\
& \cdot\left[\pi+\arctan \left(\alpha\left(\Delta \theta+\delta_{1}-\delta^{*}\right)\right)-\arctan \left(\alpha\left(\Delta \theta+\delta_{1}\right)\right)\right]
\end{aligned}
$$

and the real friction torque

$$
T_{F, l}^{\prime}=T_{F, l}+\Delta T_{F, l}\left(t, \omega_{l}\right)
$$

where all the perturbations due to parameter uncertainty $\left(\Delta K_{S}\right.$ and $\left.\Delta D_{S}\right)$ and model mismatches $\left(\Delta_{m}(t)\right.$ and $\left.\Delta T_{F, l}\left(t, \omega_{l}\right)\right)$ are bounded for bounded states for all $t \geq t_{0}$. From (51)-(53), it can be easily seen that the real interconnecting torque can be written as the torque defined in (11) and (12) perturbed by a bounded signal

$$
T_{l}^{\prime}=T_{l}+\varkappa(t),
$$

where $\varkappa(t)$, with $|\varkappa(t)| \leq K$ and $K$ being a positive finite real number, represents the effect of noise and all the uncertainties on the model (including this of friction).

It is clear from the description mentioned earlier that using the STSMO (16) and (19) (or any other asymptotic estimator for the interconnecting torque) will lead to an estimate of $T_{l}+\varkappa(t)$ instead of just $T_{l}$. This implies that the perturbation estimation error $\tilde{\phi}$ will not decay to zero, but it will converge to a compact set, specifically

$$
|\tilde{\phi}| \leq \Phi=\frac{1}{J_{l}} K .
$$

The assumption of uniform boundedness of the perturbation estimation error $\tilde{\phi}$ is not conservative considering that the states (positions and velocities) of the closed-loop system, as well as the friction torque are bounded signals and there exists no feedback of the estimation $\hat{\phi}$ to the closed-loop dynamics. It will be shown in the following that in this case, the adaptive estimator (46), (47), (49), and (50) ensures global uniform boundedness of the deadzone angle estimation error.

Proposition 1: The adaptive deadzone angle estimator defined in (46), (47), (49), and (50), where the perturbation estimation error $\tilde{\phi}=\left(1 / J_{l}\right) T_{l}^{\prime}-\hat{\phi}$ is UB by a positive constant real number $\Phi$, ensures that the trajectories of the deadzone angle estimation error $\tilde{\delta}(t)$ are uniformly globally bounded (UGB). Moreover, as $t \rightarrow \infty$, the bound is proportional to the perturbation estimation error bound $\Phi$.

Proof: The ISS property of the system (27) with respect to the perturbing input $\tilde{\phi}$ implies:

$$
|\tilde{\delta}(t)| \leq \beta_{1}\left(\left|\tilde{\delta}\left(t_{0}\right)\right|, t-t_{0}\right)+\beta_{2}\left(\sup _{0 \leq \tau \leq t} \tilde{\phi}(\tau)\right) \quad \forall|\tilde{\delta}| \geq r(|\tilde{\phi}|)
$$

where $r(|\tilde{\phi}|)=R|\tilde{\phi}|, \beta_{1}: \mathbb{R}_{\geq 0} \times \mathbb{R}_{\geq 0} \rightarrow \mathbb{R}_{\geq 0}$ is a class- $\mathcal{K} \mathcal{L}$ function, and $\beta_{2}: \mathbb{R}_{\geq 0} \rightarrow \mathbb{R}_{\geq 0}$ is a class- $\mathcal{K}$ function (see Definitions 3 and 4), with $\beta_{2}$ calculated as in the following [38, Th. 4.19]:

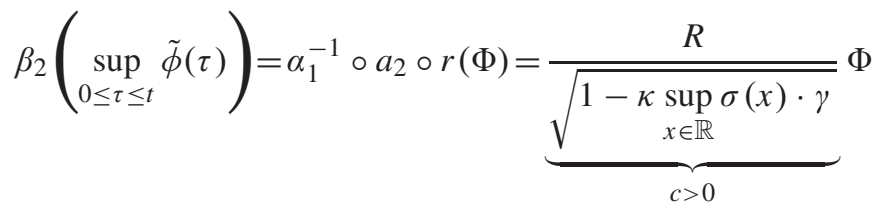

where $R$ was defined in (43).

This implies that there exists an arbitrarily large positive real number $\zeta$, such that

$$
\forall \eta \in(0, \zeta) \exists v=v(\eta)
$$

for which it holds

$$
\left|\tilde{\delta}\left(t_{0}\right)\right| \leq \eta \Rightarrow|\tilde{\delta}(t)| \leq v \quad \forall t \geq t_{0} .
$$




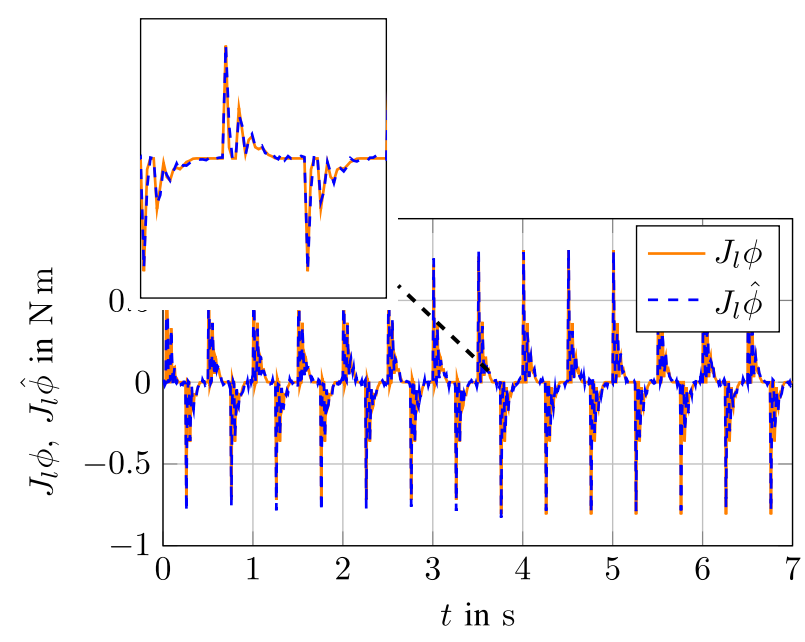

Fig. 7. Real and estimated backlash torque scaled by the load inertia $J_{l}$.

Then, according to [38, Definition 4.6], the solutions of an unperturbed deadzone estimation error system are UGB. Moreover, since $\beta \in \mathcal{K} \mathcal{L}$, it holds that

$$
\lim _{t \rightarrow \infty}|\tilde{\delta}(t)| \leq \lim _{t \rightarrow \infty}\left(\beta\left(\left|\tilde{\delta}\left(t_{0}\right)\right|, t-t_{0}\right)+c \Phi\right)=c \Phi
$$

which completes the proof.

\section{Simulation Results}

Simulations were carried out in order to demonstrate the convergence and accuracy properties of the estimation scheme. The drive motor velocity was regulated to track a sinusoidal reference signal $\omega_{r}=4 \pi \sin (4 \pi t)$. The system parameters (motor and load friction, shaft coefficients, and motor and load inertias) were considered completely known, and the velocity measurements were inflicted with zero-mean white Gaussian noise with $\sigma_{\text {meas }}=0.0316 \mathrm{rad} \mathrm{s}^{-1}$. Emulation of the backlash phenomenon was done by using the deadzone model. The deadzone angle was initially set to $\delta^{*}=0.2 \mathrm{rad}$. An increase by a $5 \%$ step of its initial value was occurred after the first $3 \mathrm{~s}$ of the simulation. Finally, the position offset was taken $\delta_{1}=\left(\delta^{*} / 2\right)$.

In the absence of model parameters uncertainties, the STSMO estimated the perturbation $\phi$ with a substantial accuracy, as shown in 7. Fig. 8 shows the estimation of the deadzone angle and the detection of the change in its value. In both cases, convergence was achieved in less than $2 \mathrm{~s}$, while the absolute steady-state estimation error was less than $2.5 \times 10^{-4} \mathrm{rad}$. This accuracy sufficiently meets the requirement set in Problem 1.

The $\Delta \theta, J_{l} \hat{\phi}$ and $\theta_{m}, \theta_{l}$ plots in Fig. 9 illustrate the estimated deadzone width and the corresponding backlash hysteresis relation between the drive motor and load positions.

The relation between the bound $K$ for the backlash torque estimation error and the steady-state deadzone estimation absolute error was investigated with additional simulation scenarios, where only the STSMO gains $k_{1}$ and $k_{2}$ were varied. In each scenario, the peak steady-state deadzone estimation error was calculated as the MAEE over the last $T_{p}$ seconds of
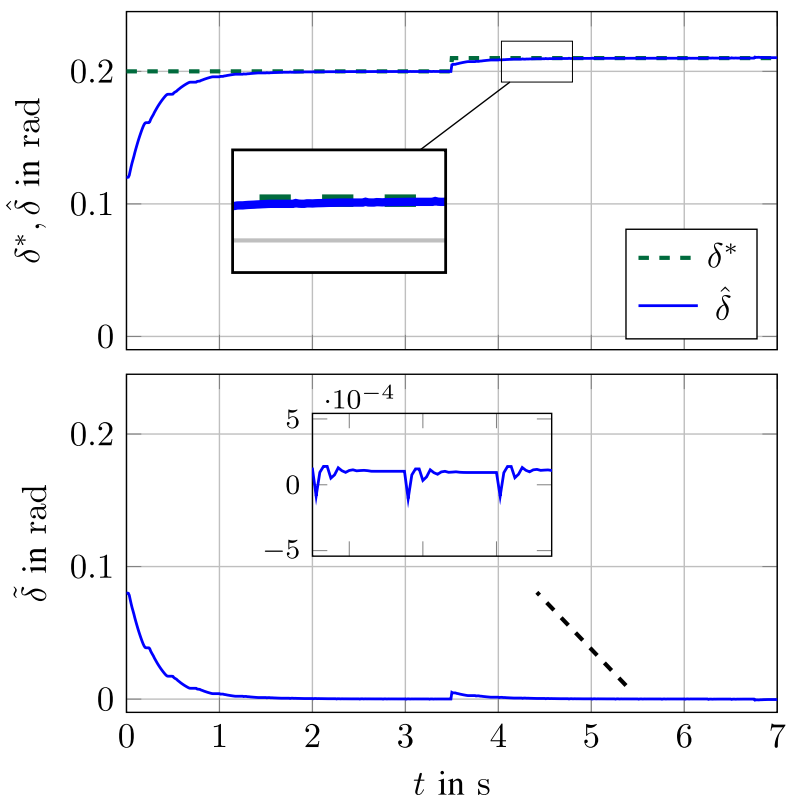

Fig. 8. Top: real and estimated change of deadzone angle. Bottom: estimation error.
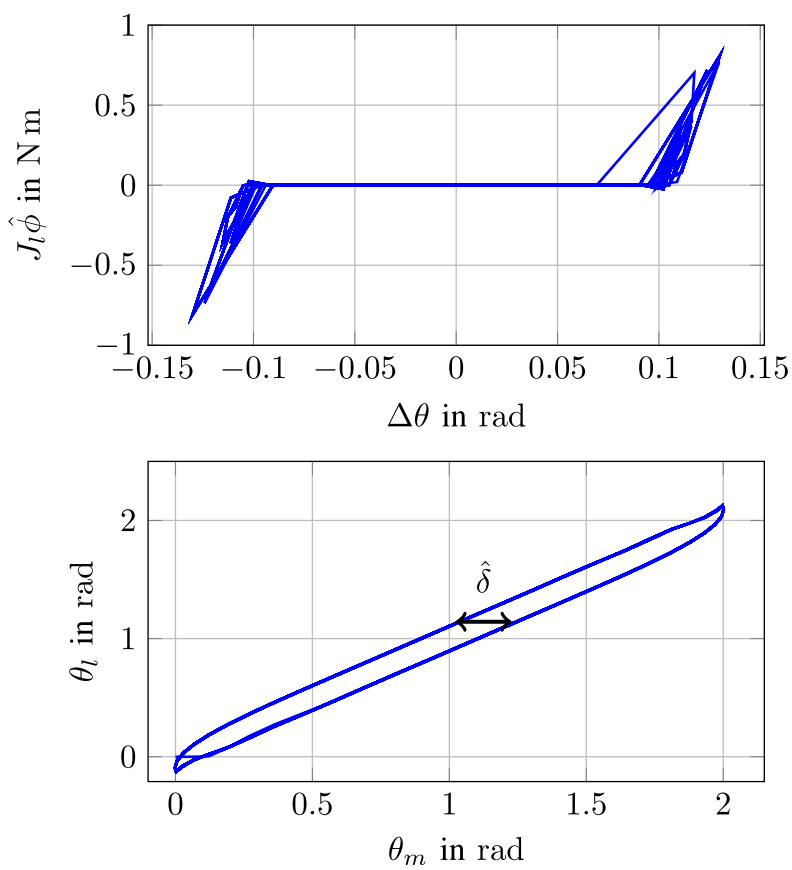

Fig. 9. Top: deadzone illustration through torque-position difference. Bottom: backlash hysteresis.

the simulation

$$
\tilde{\delta}_{\text {peak }}^{S S}=\max _{t_{f}-T_{p} \leq t \leq t_{f}}|\tilde{\delta}(t)|
$$

where $t_{f}$ denotes the end time of each simulation. The different obtained values for $\tilde{\delta}_{\text {peak }}^{S S}$ were plotted against the corresponding bounds for $J_{l}|\hat{\phi}|$. Fig. 10 shows the approximately linear relation between the two bounds with a slope $q \approx 0.0023$.

Remark 5: Although the deadzone estimation error is always bounded (due to the projection), convergence of the 


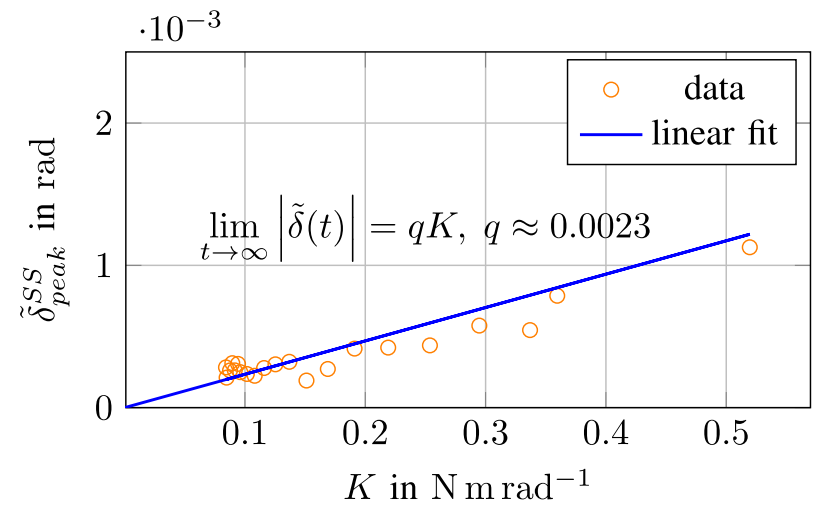

Fig. 10. Linear relation between the bounds for the perturbation estimation error $\tilde{\phi}$ and the deadzone angle estimation error $\tilde{\delta}$.

adaptive estimate $\hat{\delta}$ to the real deadzone angle is guaranteed only if a certain PE criterion, expressed in Assumption 2, is satisfied. Intuitively, this means that the reference signal should make the motor move in a way, such that substantial information on the deadzone is captured by the system measurements, i.e., the motor should engage with and disengage from the load as frequently as possible. For this reason, any palindromic motion profile, e.g., a sinusoid, is suitable for deadzone estimation experiments. The choice of the reference signal directly affects the performance of the estimator, and it may compromise it in cases where the backlash phenomenon does not appear (e.g., unidirectional axis movement with the motor and the load is already engaged).

Remark 6: The sensitivity of the estimated perturbation $\hat{\phi}$ with respect to the deadzone angle is proportional to the steepness factor $\alpha$, as can be easily seen from (46), (47), and (50). A large value for the latter, e.g., $10^{4}$, will make the estimator very sensitive to small changes in $\delta$, which is in general desirable but will set larger gain requirements for the STSMO. Indeed, selecting $k_{1}$ and $k_{2}$ based on the bound of $T_{l}$ leads to substantial chatter in the estimation signal (both $\hat{\phi}$ and $\hat{\delta}$ ). For this reason, the observer gains were iteratively tuned according to the discussion in Remark 3 . The adaptation gain $\gamma$ represents the rate of estimation of the deadzone angle. This depends on the levels of excitation, i.e., the frequency of the velocity reference signal $\omega_{r}$. Slower reference signals require larger adaptation gains if fast convergence is required. Too large gains, however, lead to more abrupt "jumps" in the estimation signal and increased sensitivity to sensor noise. As with the STSMO gains, a good practice is to start with small values for $\gamma$ and increase it until the desired convergence rate is achieved. Last, since the deadzone angle can only increase in time (due to wear of the couplings), it is natural to underestimate the initial estimation value $\hat{\delta}\left(t_{0}\right)$. Zero initial conditions are chosen if no prior knowledge about the width of the motor-to-load clearance is available.

\section{EXPERIMENTAL RESULTS}

\section{A. Experimental Setup}

The experimental setup consists of two Siemens FT70425AF70 PMSMs connected through a stainless steel shaft. Both motors are equipped with a Siemens SINAMICS S120 drive

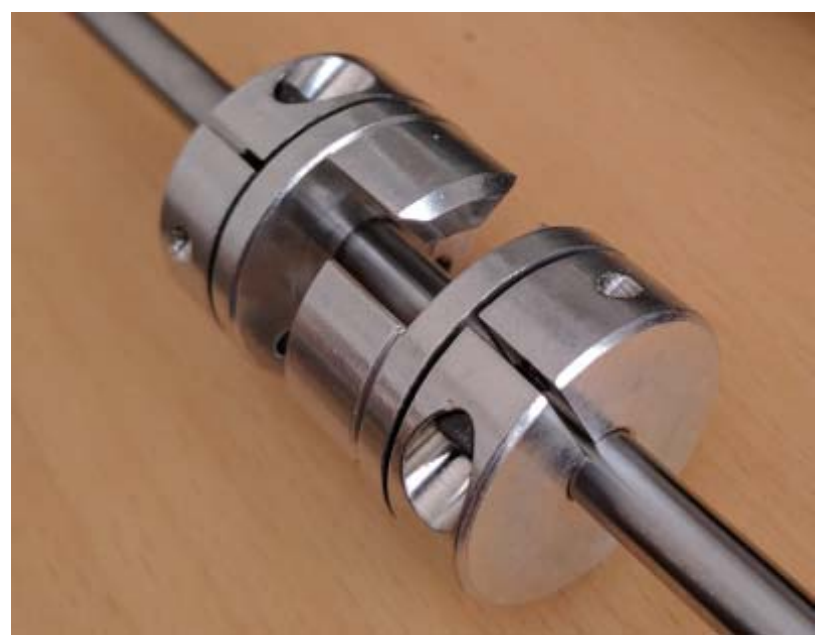

Fig. 11. Jaw coupling detail.

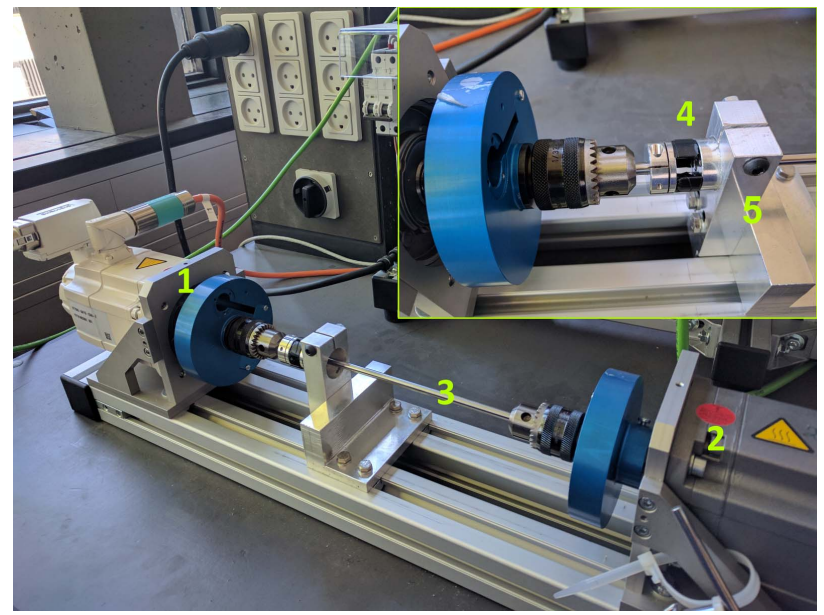

Fig. 12. Experimental setup. 1: 1FT7 drive PMSM. 2: 1FT7 load PMSM. 3: shaft. 4: backlash mechanism. 5: mounting base.

converter with 11-bit absolute encoders for the position. Three custom-made jaw couplings are mounted (one at a time) on a steel base and house the two parts of the interconnecting shaft (see Fig. 11). The total deadzone angle for each of the couplings is $1.027,0.186$, and $0.105 \mathrm{rad}\left(58.8445^{\circ}, 10.6685^{\circ}\right.$, and $6.0161^{\circ}$, respectively). The entire drive train with the friction component is shown in Fig. 12.

The load motor (see Fig. 12) serves as a pure inertia in the experiments. The design and tuning of the position, velocity, and current control loops in the drive motor were not included in this paper.

\section{B. Test Scenarios}

The identified parameters of the physical system were used in the experiments for the assessment of the estimation algorithm. Five more variation cases were considered for testing the robustness and performance of the deadzone estimator against parameter uncertainties. In each of these additional tests, one of the four uncertain parameters (shaft and friction coefficients) was increased by $100 \%$ of their identified value. In the fifth test, all four parameters $\left(K_{S}, D_{S}, T_{C, l}\right.$, and $\left.\beta_{l}\right)$ 
TABLE II

TEST SCENARIOS

\begin{tabular}{cccl} 
No & $\delta^{*}(\mathrm{rad})$ & $\delta_{1}(\mathrm{rad})$ & Parameter variation \\
\hline $0 A$ & 1.027 & 0.8924 & No variation \\
$1 A$ & 1.027 & 0.8924 & $100 \%$ increase in $K_{S}$ \\
$2 A$ & 1.027 & 0.8924 & $100 \%$ increase in $D_{S}$ \\
$3 A$ & 1.027 & 0.8924 & $100 \%$ increase in $T_{C, l}$ \\
$4 A$ & 1.027 & 0.8924 & $100 \%$ increase in $\beta_{l}$ \\
$5 A$ & 1.027 & 0.8924 & All variations combined \\
$0 B$ & 0.186 & 0 & No variation \\
$1 B$ & 0.186 & 0 & $100 \%$ increase in $K_{S}$ \\
$2 B$ & 0.186 & 0 & $100 \%$ increase in $D_{S}$ \\
$3 B$ & 0.186 & 0 & $100 \%$ increase in $T_{C, l}$ \\
$4 B$ & 0.186 & 0 & $100 \%$ increase in $\beta_{l}$ \\
$5 B$ & 0.186 & 0 & All variations combined \\
$0 C$ & 0.105 & 0.0021 & No variation \\
$1 C$ & 0.105 & 0.0021 & $100 \%$ increase in $K_{S}$ \\
$2 C$ & 0.105 & 0.0021 & $100 \%$ increase in $D_{S}$ \\
$3 C$ & 0.105 & 0.0021 & $100 \%$ increase in $T_{C, l}$ \\
$4 C$ & 0.105 & 0.0021 & $100 \%$ increase in $\beta_{l}$ \\
$5 C$ & 0.105 & 0.0021 & All variations combined \\
\hline
\end{tabular}

were increased simultaneously. The reference for the position of the drive motor was a sinusoid

$$
\theta_{r}(t)=\Theta_{0} \sin \left(2 \pi f_{r} t\right)
$$

where in all the tests, $\Theta_{0}=1 \mathrm{rad}$ and $f_{r}=0.5 \mathrm{~Hz}$. The 15 different scenarios are shown in Table II. The parameters of the identified physical system and the SMO and adaptive estimator are collectively given in Table III.

\section{Results}

This section presents the results obtained from the experiments on the estimation of the deadzone angle. The performance of the estimator was evaluated based on the maximum absolute estimation error (MAEE), which is defined over a time interval $T>0$ as

$$
\text { MAEE }=\sup _{t_{0} \leq t \leq t_{0}+T}|\tilde{\delta}(t)| .
$$

Fig. 13 shows the estimation of the deadzone angle for the cases where the identified parameters of the system were used (Tests $0 \mathrm{~A}, 0 \mathrm{~B}$, and $0 \mathrm{C}$ ). As it can be seen, the estimation error $\tilde{\delta}$ converges to a compact set including the origin, while the corresponding MAEE is less than $10^{-2} \mathrm{rad}$ in all the tests, specifically $1.75 \times 10^{-3}, 1.90 \times 10^{-3}$, and $0.66 \times 10^{-3} \mathrm{rad}$ for Tests $0 \mathrm{~A}, 0 \mathrm{~B}$, and $0 \mathrm{C}$, respectively.

The position of the motor and the load can be seen in Fig. 14 (top). While in the deadzone, the load does not move although the drive motor shaft does. This loss of engagement is also shown in the bottom of Fig. 14, where the connecting
TABLE III

\begin{tabular}{|c|c|c|}
\hline Symbol & Description & Value \\
\hline \multicolumn{3}{|c|}{ STSMO parameters } \\
\hline$k_{1}$ & Switching gain & 0.5 \\
\hline$k_{2}$ & Integral switching gain & 70 \\
\hline \multicolumn{3}{|c|}{ Estimator parameters } \\
\hline$\alpha$ & Stiffness model slope & $10^{4}$ \\
\hline$\gamma$ & Adaptation gain & $5 \cdot 10^{-3}$ \\
\hline$\hat{\delta}_{0}$ & Initial conditions & $0 \mathrm{rad}$ \\
\hline$\delta_{\max }$ & Maximum deadzone & $2 \pi \mathrm{rad}$ \\
\hline \multicolumn{3}{|c|}{ Load system parameters } \\
\hline$J_{l}$ & Inertia & $0.000831 \mathrm{~kg} \mathrm{~m}^{2}$ \\
\hline$K_{S}$ & Shaft stiffness & $31.75 \mathrm{~N} \mathrm{~m} \mathrm{rad}^{-1}$ \\
\hline$D_{S}$ & Shaft damping coefficient & $0.054 \mathrm{~N} \mathrm{~m} \mathrm{~s} \mathrm{rad}^{-1}$ \\
\hline$N$ & Gearing ratio & 1 \\
\hline$T_{C, l}$ & Coulomb friction & $0.0792 \mathrm{~N} \mathrm{~m}$ \\
\hline$\beta_{l}$ & Viscous friction coefficient & $0.031 \mathrm{~N} \mathrm{~ms} \mathrm{rad}^{-1}$ \\
\hline
\end{tabular}

System and Estimator Parameters' Values
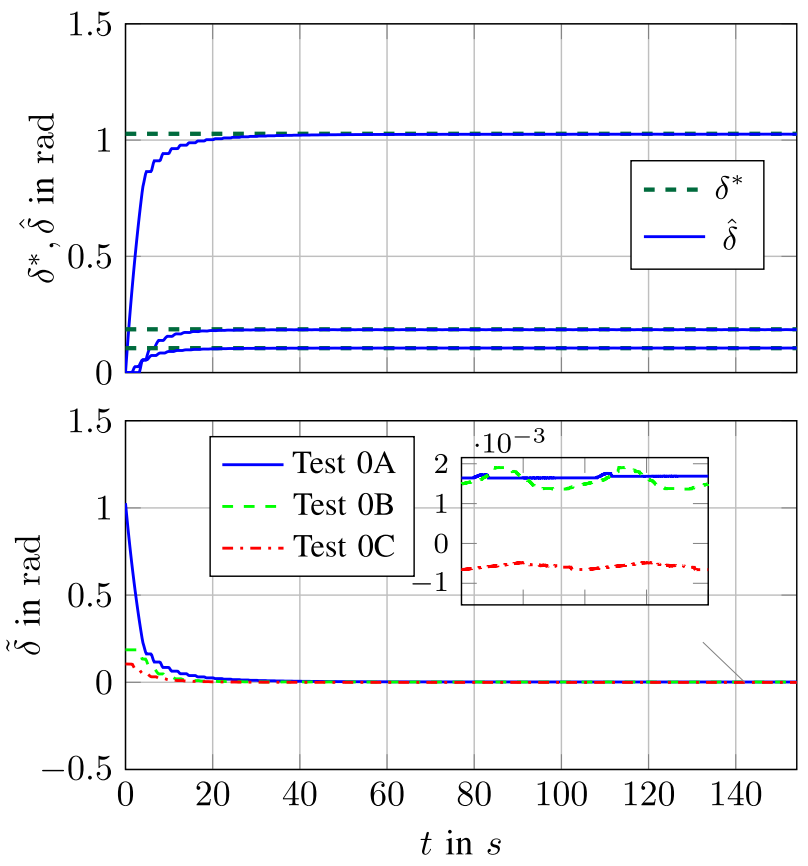

Fig. 13. Deadzone angle estimated value (top) and the associated estimation error (bottom) for the nominal cases (Tests $0 \mathrm{~A}, 0 \mathrm{~B}$, and $0 \mathrm{C}$ ).

shaft stiffness is depicted. Whenever the system is in the deadzone, the shaft stiffness is 0 . During the initial part of the motion in the positive direction, the stiffness is 0 even though the load is moving. This is due to the fact that impact torque exerted by the drive motor on the load temporarily accelerates the load, making it move even though there is no actual engagement.

An illustration of the estimated deadzone is given in Fig. 15, where the backlash torque $J_{l} \hat{\phi}$ estimate and the equivalent 

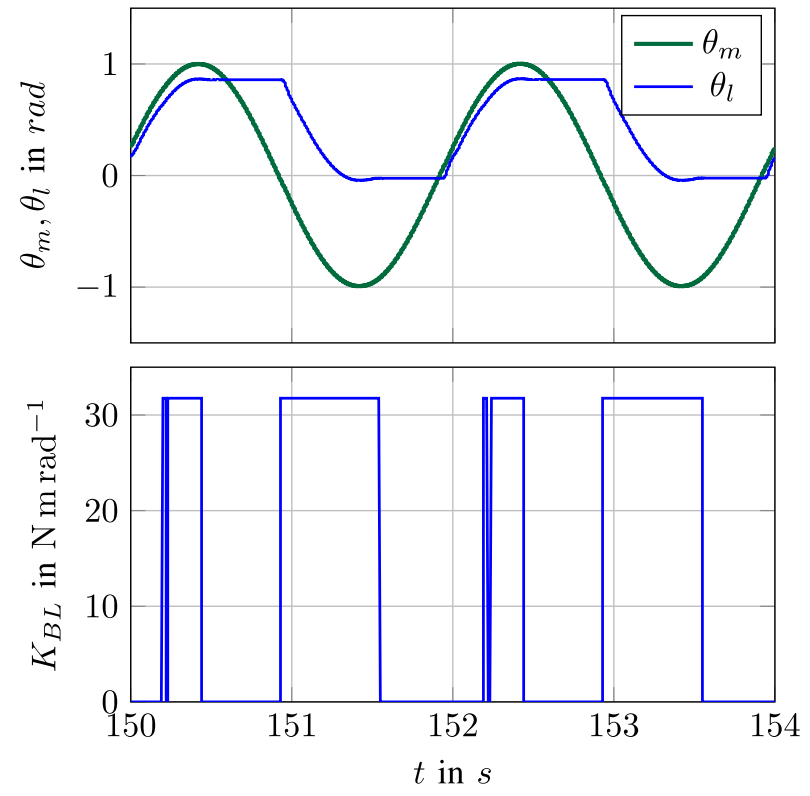

Fig. 14. Motor and load position (top) and the calculated shaft stiffness (bottom) for the nominal case with $\delta^{*}=1.027 \mathrm{rad}$ (Test $0 \mathrm{~A}$ ).
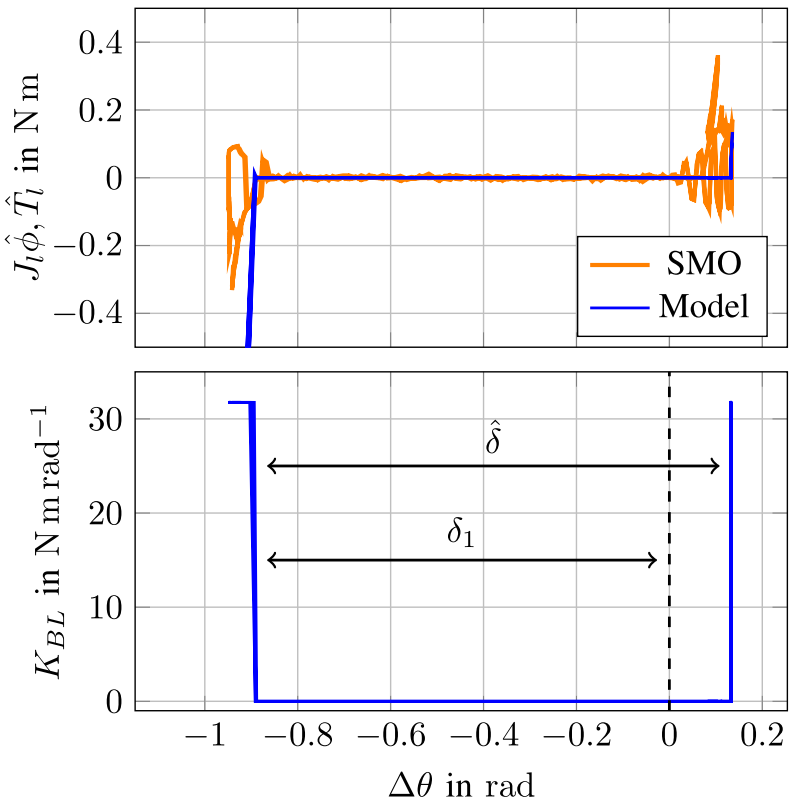

Fig. 15. Deadzone illustration through torque-position difference (top) and torque-stiffness (bottom) plots during Test 0A. The deadzone is not symmetric around $\Delta \theta=0$ but offset due to the initial position difference $\delta_{1}$, specifically by $\left(\delta^{*} / 2\right)-\delta_{1}$.

calculation based on the stiffness model (top) are depicted as functions of the position difference between motor and load. The oscillations at the two ends of the deadzone in the estimated torque correspond to the impact between motor and load upon engagement. Fig. 15 (bottom) shows the stiffness $K_{\mathrm{BL}}$ also as a function of $\Delta \theta$.

The backlash hysteresis can be seen in the top of Fig. 16, which shows $4 \mathrm{~s}$ of the time response of the load position with respect to the motor. Fig. 16 (bottom) shows the drive motor and load phase portraits, i.e., the plots of their angular velocities with respect to their positions.
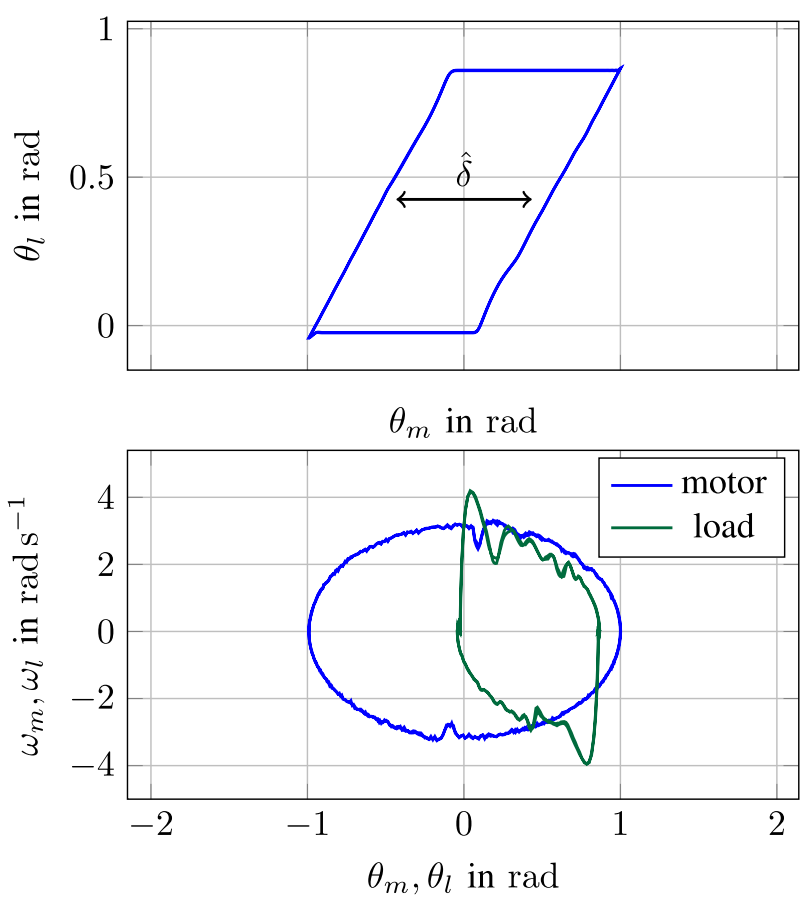

Fig. 16. Backlash hysteresis (top) and phase portraits for the motor and load (bottom) during Test $0 \mathrm{~A}$.
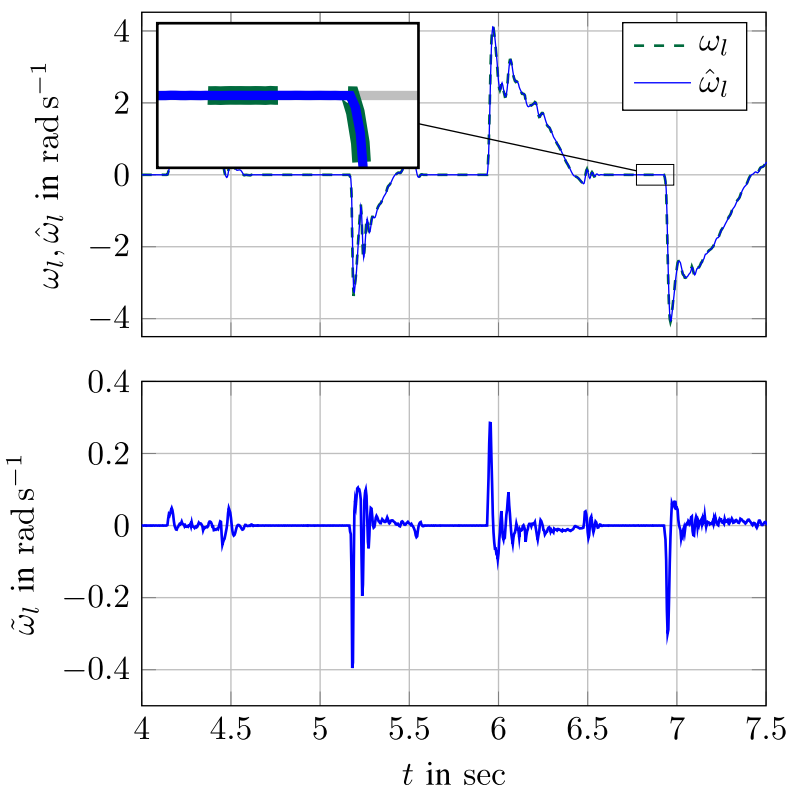

Fig. 17. Real and estimated load velocity (top) and the associated estimation error (bottom) during Test 0A.

Fig. 17 shows the actual and estimated by the SMO load angular velocity during Test 0A. As it can be seen in Fig. 17 (bottom), the velocity estimation error is smaller than $0.4 \mathrm{rad} \mathrm{s}^{-1}$ in magnitude. Such an error is sufficiently small, so that the estimated backlash torque, shown in Fig. 18, can be considered accurate enough to be used in the deadzone estimation algorithm.

The deadzone angle estimation error for all the parameter variations is shown in Fig. 19 (top for Tests 1A-5A, middle 


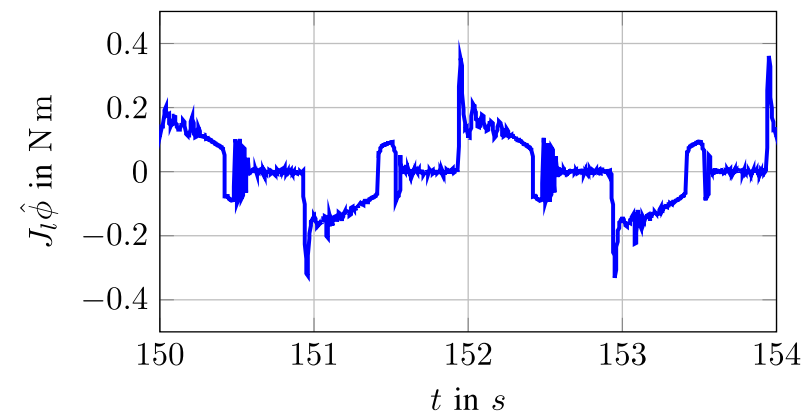

Fig. 18. Estimated backlash torque for the nominal case with $\delta^{*}=1.027 \mathrm{rad}$ (Test 0A).
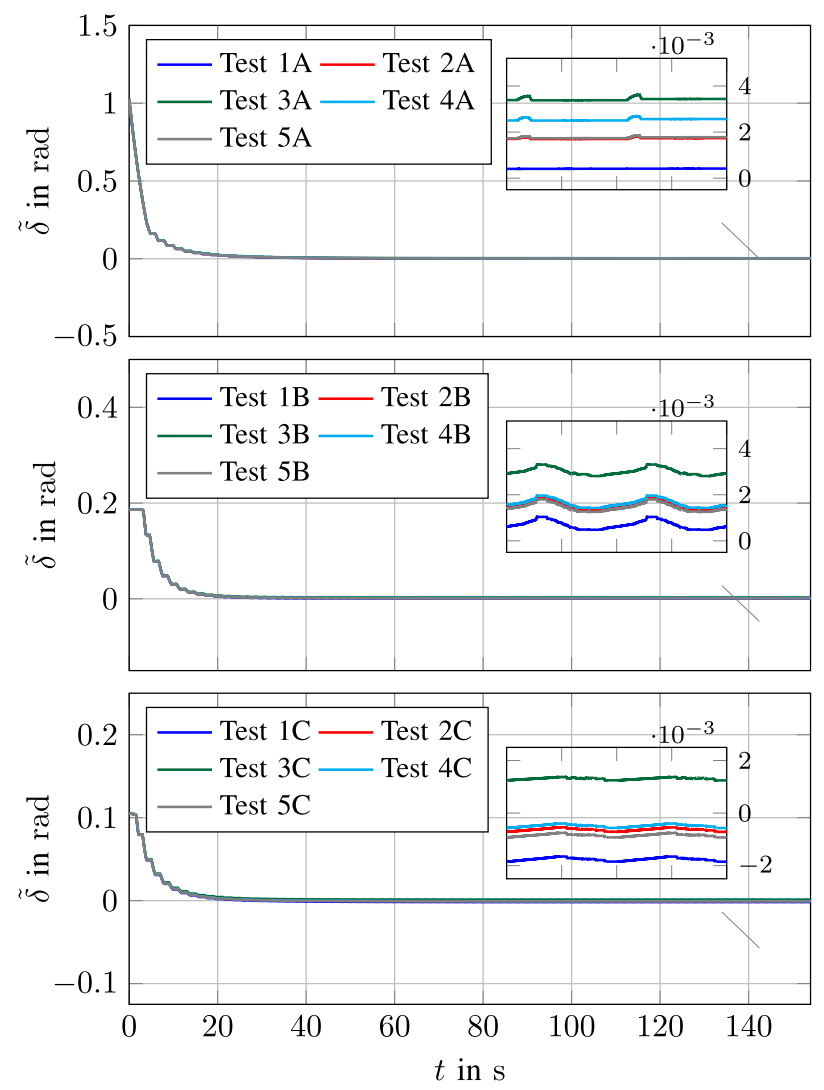

Fig. 19. Deadzone angle estimation error during Tests $1 \mathrm{~A}-5 \mathrm{~A}$ (top), Tests 1B-5B (middle), and Tests 1C-5C 4 (bottom).

for Tests $1 \mathrm{~B}-5 \mathrm{~B}$, and bottom for Tests $1 \mathrm{C}-5 \mathrm{C})$. As it can be seen, in all the considered cases, the estimates converge to a constant value sufficiently close to the real one. Specifically, all the estimation errors do not exceed $4 \times 10^{-3}$ rad in magnitude, which implies more than $40 \%$ better performance from that required in Problem 1. The MAEE during all Tests is presented in Table IV.

Remark 7: The sudden engagement between drive motor and load has produced a periodic impact torque acting on both parts. This caused a small displacement of the load, which resulted in a shift of the position offset $\delta_{1}$. However, the effect of this change in the offset on the quality of the estimation was practically negligible.
TABLE IV

MAEE In mrad For the Nominal CASE (Tests 0A, 0B, AND 0C) AND All the Parameter Variations (Tests 1A-5A, 1B-5B, and 1C-5C)

\begin{tabular}{ccccccc}
\hline$\delta^{*}$ in rad & 0 & 1 & 2 & 3 & 4 & 5 \\
\hline 1.027 (case "A") & 1.75 & 0.41 & 1.80 & 3.61 & 2.69 & 1.85 \\
0.186 (case "B") & 1.90 & 1.04 & 1.87 & 3.31 & 1.96 & 1.80 \\
0.105 (case "C") & 0.66 & 1.85 & 0.72 & 1.38 & 0.57 & 0.93 \\
\hline
\end{tabular}

\section{CONCLUSION}

A deadzone angle estimation method for a single-axis drive train with backlash was presented in this paper. The design is a modified version of the cascaded SMO-adaptive estimator scheme presented in [9], which now includes an initial position offset between drive motor and load. The stability and robustness properties of the algorithm were discussed in the context of model and parameter uncertainties. Specifically, it was proven that the estimation error is UGAS in the absence of model and parameter mismatches and UGB otherwise with the bound being proportional to the total perturbation estimation error bound.

The theoretical findings were experimentally validated on a real single-axis drive train with the state-of-the-art Siemens equipment for the cases where the deadzone was equal to 1.027, 0.186, and $0.105 \mathrm{rad}$. The method was tested both for the identified system parameters and for the case of $100 \%$ offset in each of these parameters. In all the tests, convergence was achieved in approximately $40 \mathrm{~s}$ and with precision in the order of $10^{-3} \mathrm{rad}$.

Future work will include the assessment of the algorithm performance in estimating even smaller angles and also the design of a joint estimation scheme for both the deadzone angle and the initial position offset.

\section{APPENDIX A \\ BASIC DEFINITIONS}

Definition 1 (PE [39]): A piecewise continuous signal vector $\phi: \mathbb{R}^{+} \rightarrow \mathbb{R}^{n}$ is persistently exciting with a level of excitation $\alpha_{0}>0$ if there exist constants $\alpha_{1}, T_{0}>0$ such that

$$
\alpha_{0} I \leq \frac{1}{T_{0}} \int_{t}^{t+T_{0}} \boldsymbol{\phi}(\tau) \boldsymbol{\phi}^{T}(\tau) d \tau \leq \alpha_{1} I
$$

where $I$ is the $n \times n$ unity matrix.

Definition 2 (Signum Function): The signum function $\operatorname{sgn}(\cdot)$ is defined as

$$
\operatorname{sgn}(y)= \begin{cases}1 & \text { if } y>0 \\ v \in[-1,1] & \text { if } y=0 \\ -1 & \text { if } y<0 .\end{cases}
$$

Definition $3\left(\mathcal{K}, \mathcal{K}_{\infty} \quad\right.$ Functions [38, Definition 4.2]): A continuous function $\alpha:[0, a) \rightarrow(0, \infty]$ is said to belong to class- $\mathcal{K}$ if it is strictly increasing and $\alpha(0)=0$. It is said to belong to class- $\mathcal{K}_{\infty}$ if $a=\infty$ and $\alpha(r) \rightarrow \infty$ as $r \rightarrow \infty$. 
Definition $4(\mathcal{K} \mathcal{L}$ Functions [38, Definition 4.3]): A continuous function $\beta:[0, a) \times(0, \infty] \rightarrow(0, \infty]$ is said to belong to class- $\mathcal{K} \mathcal{L}$ if, for each fixed $s$, the mapping $\beta(s, r)$ belongs to class- $\mathcal{K}$ with respect to $r$ and, for each fixed $r$, the mapping $\beta(r, s)$ is decreasing with respect to $s$ and $\beta(r, s) \rightarrow 0$ as $s \rightarrow \infty$.

Definition 5 (Input-to-State Stability [38, Definition 4.7]): A system of the form

$$
\dot{x}=f(t, x, u)
$$

is said to be ISS with respect to the input $\boldsymbol{u}$ if there exist a class- $\mathcal{K} \mathcal{L}$ function $\beta$ and a class- $\mathcal{K}$ function $\gamma$, such that for any initial state $\boldsymbol{x}\left(t_{0}\right)$ and any bounded input $\boldsymbol{u}(t)$, the solution $\boldsymbol{x}(t)$ exists for all $t \geq t_{0}$ and satisfies

$$
\|\boldsymbol{x}(t)\| \leq \beta\left(\left\|\boldsymbol{x}\left(t_{0}\right)\right\|, t-t_{0}\right)+\gamma\left(\sup _{t_{0} \leq \tau \leq t}\|\boldsymbol{u}(\tau)\|\right) .
$$

\section{APPENDIX B \\ PROJECTION OPERATOR}

Let $\Omega_{c}$ be a convex subset of the parameter space $\mathcal{D}$ defined as $\Omega_{l} \triangleq\{\delta \in \mathcal{D} \mid h(\delta) \leq l\}$, where $l>0$ and $h: \mathbb{R} \rightarrow \mathbb{R}$ is a smooth convex function. The projection operator is defined as follows [40]:

$\operatorname{Proj}(\hat{\delta}, \tau)= \begin{cases}\tau, & h(\hat{\delta})<0 \\ \tau, & h(\hat{\delta}) \geq 0 \& \nabla h^{T} \tau \leq 0 \\ \tau-\frac{\nabla h}{|\nabla h|}\left\langle\frac{\nabla h}{|\nabla h|}, \tau\right) h(\hat{\delta}), & h(\hat{\delta}) \geq 0 \& \nabla h^{T} \tau>0 .\end{cases}$

In this paper, the convex function $h$ has been chosen according to [40]

$$
h(\hat{\delta}) \triangleq \frac{\left(\epsilon_{\delta}+1\right) \hat{\delta}^{2}-\delta_{\max }^{2}}{\epsilon_{\delta} \delta_{\max }^{2}} .
$$

In the above definition of $h, \delta_{\max }$ is a conservative upper bound for the backlash angle estimate $\hat{\delta}$ and $\epsilon_{\delta}$ is a small positive number. The operator $\langle\cdot, \cdot\rangle$ denotes the inner product, which in this case reduces into a real product.

\section{APPENDIX C \\ TAYLOR EXPANSION}

Reference [37, Th. 11.1]: Suppose that $\boldsymbol{r}: \mathbb{R}^{n} \rightarrow \mathbb{R}^{n}$ is continuously differentiable in some convex open set $\mathbb{D}$ and that $\boldsymbol{y}$ and $\boldsymbol{y}+\boldsymbol{q}$ are vectors in $\mathbb{D}$. We then have that

$$
\boldsymbol{r}(\boldsymbol{y}+\boldsymbol{q})=\boldsymbol{r}(\boldsymbol{y})+\int_{0}^{1} \frac{\partial \boldsymbol{r}}{\partial \boldsymbol{y}}(\boldsymbol{y}+p \boldsymbol{q}) \boldsymbol{q} d p .
$$

Setting $\boldsymbol{y} \triangleq \hat{\delta}, \boldsymbol{q} \triangleq \tilde{\delta}, \boldsymbol{r} \triangleq T_{l}$, the theorem reads

$$
T_{l}(\boldsymbol{x}, \underbrace{\hat{\delta}+\tilde{\delta}}_{\delta^{*}})-T_{l}(\boldsymbol{x}, \hat{\delta})=\int_{0}^{1} \frac{\partial T_{l}}{\partial \hat{\delta}}(\boldsymbol{x}, \hat{\delta}+p \tilde{\delta}) \tilde{\delta} d p .
$$

\section{APPENDIX D \\ PROOF OF PROPERTY (33)}

For $t, T \geq 0$, it holds

$$
\begin{aligned}
\int_{0}^{\infty} e^{(t-\tau)} \sigma(x(\tau)) d \tau & \geq \int_{t}^{t+T} e^{(t-\tau)} \sigma(x(\tau)) d \tau \\
& =e^{-T} \int_{t}^{t+T} e^{(t+T-\tau)} \sigma(x(\tau)) d \tau .
\end{aligned}
$$

Moreover,

$$
t \leq \tau \leq T \Rightarrow t+T-\tau \geq 0 \Rightarrow e^{(t+T-\tau)} \geq 1
$$

leading to

$$
e^{-T} \int_{t}^{t+T} e^{(t+T-\tau)} \sigma(x(\tau)) d \tau \geq e^{-T} \int_{t}^{t+T} \sigma(x(\tau)) d \tau
$$

which proves the property.

\section{REFERENCES}

[1] J. L. Stein and C.-H. Wang, "Estimation of gear backlash: Theory and simulation," J. Dyn. Syst., Meas., Control, vol. 120, no. 1, pp. 74-82, 1998.

[2] G. Hovland, S. Hanssen, S. Moberg, T. Brogårdh, S. Gunnarsson, and M. Isaksson, "Nonlinear identification of backlash in robot transmissions," in Proc. 33rd ISR Int. Symp. Robot., Oct. 2002, pp. 3-8.

[3] A. Lagerberg and B. S. Egardt, "Estimation of backlash with application to automotive powertrains," in Proc. 42nd IEEE Conf. Decision Control, vol. 5, Dec. 2003, pp. 4521-4526.

[4] T. Hägglund, "Automatic on-line estimation of backlash in control loops," J. Process Control, vol. 17, no. 6, pp. 489-499, 2007.

[5] J. Vörös, "Modeling and identification of systems with backlash," Automatica, vol. 46, no. 2, pp. 369-374, 2010.

[6] P. Templin, "Simultaneous estimation of driveline dynamics and backlash size for control design," in Proc. IEEE Int. Conf. Control Appl., Sep. 2008, pp. 13-18.

[7] R. Merzouki and J. C. Cadiou, "Estimation of backlash phenomenon in the electromechanical actuator," Control Eng. Pract., vol. 13, no. 8, pp. 973-983, 2005.

[8] R. Merzouki, J. A. Davila, L. Fridman, and J. C. Cadiou, "Backlash phenomenon observation and identification in electromechanical system," Control Eng. Pract., vol. 15, no. 4, pp. 447-457, 2007.

[9] D. Papageorgiou, M. Blanke, H. H. Niemann, and J. H. Richter, "Backlash estimation for industrial drive-train systems," IFAC-PapersOnLine, vol. 50, no. 1, pp. 3281-3286, 2017.

[10] O. Egeland and J. T. Gravdahl, Modeling and Simulation for Automatic Control, vol. 76. Trondheim, Norway: Marine Cybernetics, 2002.

[11] M. Nordin, J. Galic', and P.-O. Gutman, "New models for backlash and gear play," Int. J. Adapt. Control Signal Process., vol. 11, no. 1, pp. 49-63, 1997.

[12] R. Kalantari and F. M. Sadat, "Backlash nonlinearity modeling and adaptive controller design for an electromechanical power transmission system," Sci. Iranica, vol. 16, no. 6, pp. 463-469, 2009.

[13] H. Logemann and E. P. Ryan, "Systems with hysteresis in the feedback loop: Existence, regularity and asymptotic behaviour of solutions," ESAIM, Control, Optim. Calculus Variat., vol. 9, pp. 169-196, Mar. 2003.

[14] B. Jayawardhana, H. Logemann, and E. P. Ryan, "PID control of second-order systems with hysteresis," Int. J. Control, vol. 81, no. 8, pp. 1331-1342, 2008.

[15] H. Logemann, E. P. Ryan, and I. Shvartsman, "A class of differentialdelay systems with hysteresis: Asymptotic behaviour of solutions," Nonlinear Anal., Theory, Methods Appl., vol. 69, no. 1, pp. 363-391, 2008.

[16] J. C. Gerdes and V. Kumar, "An impact model of mechanical backlash for control system analysis," in Proc. IEEE Amer. Control Conf., vol. 5, 1995, pp. 3311-3315.

[17] J. A. de Marchi, "Modeling of dynamic friction, impact backlash and elastic compliance nonlinearities in machine tools, with applications to asymmetric viscous and kinetic friction identification," Ph.D. dissertation, Rensselaer Polytech. Inst., Troy, NY, USA, 1998. 
[18] G. Tao and P. V. Kokotović, "Adaptive control of systems with backlash," Automatica, vol. 29, no. 2, pp. 323-335, Mar. 1993.

[19] M. Nordin and P.-O. Gutman, "Controlling mechanical systems with backlash-A survey," Automatica, vol. 38, no. 10, pp. 1633-1649, 2002.

[20] C. Cao, A. M. Annaswamy, and A. Kojic, "Parameter convergence in nonlinearly parameterized systems," IEEE Trans. Autom. Control, vol. 48, no. 3, pp. 397-412, Mar. 2003.

[21] I. Y. Tyukin, D. V. Prokhorov, and C. V. Leeuwen, "Adaptation and parameter estimation in systems with unstable target dynamics and nonlinear parametrization," IEEE Trans. Autom. Control, vol. 52, no. 9 , pp. 1543-1559, Sep. 2007.

[22] H. F. Grip, A. Saberi, and T. A. Johansen, "Estimation of states and parameters for linear systems with nonlinearly parameterized perturbations," Syst. Control Lett., vol. 60, no. 9, pp. 771-777, 2011.

[23] H. F. Grip, A. Saberi, and T. A. Johansen, "State and parameter estimation for nonlinearly parameterized systems: An $H_{\infty}$-based approach," IFAC Proc. Vol., vol. 44, no. 1, pp. 2997-3002, 2011.

[24] I. Y. Tyukin, E. Steur, H. Nijmeijer, and C. van Leeuwen, "Adaptive observers and parameter estimation for a class of systems nonlinear in the parameters," Automatica, vol. 49, no. 8, pp. 2409-2423, 2013.

[25] I. Y. Tyukin, P. Rogachev, and H. Nijmeijer, "Adaptive observers for nonlinearly parameterized systems subjected to parametric constraints," IFAC Proc. Vol., vol. 47, no. 3, pp. 10869-10874, 2014.

[26] H. F. Grip, T. A. Johansen, L. Imsland, and G.-O. Kaasa, "Parameter estimation and compensation in systems with nonlinearly parameterized perturbations," Automatica, vol. 46, no. 1, pp. 19-28, 2010.

[27] S. K. Spurgeon, "Sliding mode observers: A survey," Int. J. Syst. Sci., vol. 39, no. 8, pp. 751-764, 2008.

[28] Y. Shtessel, C. Edwards, L. Fridman, and A. Levant, Sliding Mode Control and Observation. New York, NY, USA: Springer, 2014.

[29] A. Levant, "Sliding order and sliding accuracy in sliding mode control," Int. J. Control, vol. 58, no. 6, pp. 1247-1263, Dec. 1993.

[30] J. Davila, L. M. Fridman, and A. Levant, "Second-order sliding-mode observer for mechanical systems," IEEE Trans. Autom. Control, vol. 50, no. 11, pp. 1785-1789, Nov. 2005.

[31] J. A. Moreno and M. Osorio, "Strict Lyapunov functions for the super-twisting algorithm," IEEE Trans. Autom. Control, vol. 57, no. 4, pp. 1035-1040, Apr. 2012.

[32] L. Fridman, J. Moreno, and R. Iriarte, Eds., Sliding Modes After the First Decade of the 21st Century (Lecture Notes in Control and Information Sciences). Berlin, Germany: Springer, 2012.

[33] G. Bartolini, A. Ferrara, E. Usai, and V. I. Utkin, "On multi-input chattering-free second-order sliding mode control," IEEE Trans. Autom. Control, vol. 45, no. 9, pp. 1711-1717, Sep. 2000.

[34] A. Chalanga and F. Plestan, "Finite time stabilization of an uncertain chain of integrators by integral sliding mode approach," IFACPapersOnLine, vol. 50, no. 1, pp. 9613-9618, 2017.

[35] A. Loría and E. Panteley, "Cascaded nonlinear time-varying systems: Analysis and design," in Advanced Topics in Control Systems Theory (Lecture Notes in Control and Information Science), vol. 311. London, U.K.: Springer, 2005, pp. 23-64.

[36] M. Krstic, I. Kanellakopoulos, and P. V. Kokotovic, Nonlinear and Adaptive Control Design, 1st ed. Hoboken, NJ, USA: Wiley, 1995.

[37] S. Wright and J. Nocedal, Numerical Optimization, vol. 35. New York, NY, USA: Springer-Verlag, 1999, pp. 67-68.

[38] H. Khalil, Nonlinear Systems. Englewood Cliffs, NJ, USA: Prentice-Hall, 2002.

[39] P. Ioannou and J. Sun, Robust Adaptive Control (Dover Books on Electrical Engineering). New York, NY, USA: Dover, Nov./Dec. 2012.

[40] N. Hovakimyan and C. Cao, L1 Adaptive Control Theory: Guaranteed Robustness With Fast Adaptation (Advances in Design and Control). Philadelphia, PA, USA: SIAM, 2010.

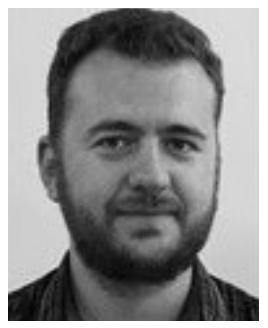

Dimitrios Papageorgiou received the Diploma degree in electrical and computer engineering from the National Technical University of Athens, Athens, Greece, in 2011, and the M.Sc. degree in electrical engineering (majoring in automation and robotics technology) and the Ph.D. degree from the Technical University of Denmark (DTU), Kongens Lyngby, Denmark, in 2014 and 2017, respectively.

$\mathrm{He}$ is currently a Post-Doctoral Researcher with the Automation and Control Group, Electrical Engineering Department, DTU. His current research interests include nonlinear and adaptive control and estimation of electromechanical systems.

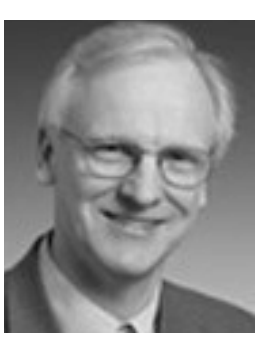

Mogens Blanke (M'74-SM'85) received the M.Sc.E.E. and Ph.D. degrees from the Technical University of Denmark (DTU), Kongens Lyngby, Denmark, in 1974 and 1982, respectively.

He was a Systems Analyst with the European Space Agency, Enschede, The Netherlands, from 1975 to 1976 and DTU from 1977 to 1984 . He was the Head of the Division, Lyngs $\varnothing$ Marine, Hørsholm, Denmark, from 1985 to 1989, and a Professor with Aalborg University, Aalborg, Denmark, from 1990 to 1999 . He has been a Professor in automation and control with DTU since 2000. He was an Adjunct Professor with the Norwegian University of Science and Technology, Trondheim, Norway, from 2005 to 2018. His current research interests include diagnosis, prognosis, and fault-tolerant control.

Dr. Blanke is a member of the International Federation of Automatic Control (IFAC) SAFEPROCESS and the Technical Committee (TC) on Marine Systems. He has held various positions at IFAC, including the Chair of the TC on Marine Systems and the CC Chair. He was a Technical Editor of the IEEE TRANSACTIONS OF AERospace and EleCtronic Systems from 2006 to 2016. He is an Associate Editor of Control Engineering Practice.

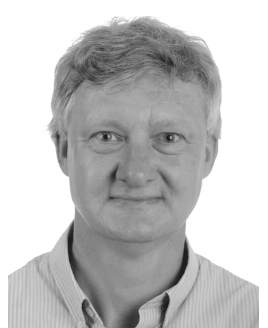

Hans Henrik Niemann was born in Slagelse, Denmark in 1961. He received the M.Sc. degree in mechanical engineering and the Ph.D. degree from the Technical University of Denmark, Kongens Lyngby, Denmark, in 1986 and 1988, respectively.

From 1988 to 1994, he held a research position at the Technical University of Denmark, where he has been an Assistant Professor in control engineering since 1994 . He is the first author in over 80 journals and conferences papers. His current research interests include optimal and robust control, fault detection and isolation, active fault diagnosis, fault-tolerant control, controller architecture for controller switching and fault-tolerant control, system and performance monitoring, and controller antiwindup.

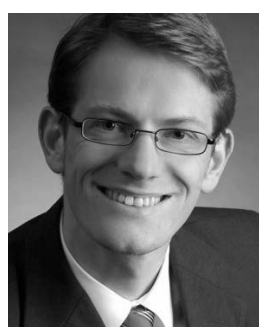

Jan H. Richter received the Diploma degree in electrical engineering from the Technische Universität Hamburg, Hamburg, Germany, in 2004, and the $\mathrm{Ph} . \mathrm{D}$. degree in control theory from Ruhr-Universität Bochum, Bochum, Germany, in 2009.

$\mathrm{He}$ is currently an Expert in control, a Project Manager, and a Researcher with the Digital Factory Division, Siemens AG, Nuremberg, Germany. His current research interests include the tool chain for model-based systems engineering, model-based controller design, verification and validation, and faulttolerant control in embedded and factory automation applications. 